\title{
A Systematic Review on the Prevalence of Overweight and Obesity, in Iranian Children and Adolescents
}

\author{
Shirin Djalalinia, ${ }^{1,2}$ Roya Kelishadi, ${ }^{3}$ Mostafa Qorbani, ${ }^{4}$ Niloofar Peykari,, ${ }^{1,2}$ Amir Kasaeian, ${ }^{1,5}$ Ensieh \\ Nasli-Esfahani, ${ }^{6}$ Shohreh Naderimagham, ${ }^{1}$ Bagher Larijani, ${ }^{7}$ and Farshad Farzadfar ${ }^{1,7,}$ \\ ${ }^{1}$ Non-Communicable Diseases Research Center, Endocrinology and Metabolism Research Institute, Tehran University of Medical Sciences, Tehran, IR Iran \\ ${ }^{2}$ Development of Research and Technology Center, Deputy of Research and Technology, Ministry of Health and Medical Education, Tehran, IR Iran \\ ${ }^{3}$ Child Growth and Development Research Center, Isfahan University of Medical Sciences, Isfahan, IR Iran \\ ${ }^{4}$ Department of Community Medicine, School of Medicine, Alborz University of Medical Sciences, Karaj, IR Iran \\ ${ }^{5}$ Department of Epidemiology and Biostatistics, School of Public Health, Tehran University of Medical Sciences, Tehran, IR Iran \\ ${ }^{6}$ Diabetes Researcher Center, Endocrinology and Metabolism Clinical Sciences Institute, Tehran University of Medical Sciences, Tehran, IR Iran \\ ${ }^{7}$ Endocrinology and Metabolism Research Center, Endocrinology and Metabolism Research Institute, Tehran University of Medical Sciences, Tehran, IR Iran \\ "Corresponding author: Farshad Farzadfar, Endocrinology and Metabolism Research Center, Endocrinology and Metabolism Research Institute, Tehran University of Medical \\ Sciences, P. O. Box: 1599666615, Tehran, IR Iran. Tel/Fax: +98-2188913543, E-mail: f-farzadfar@tums.ac.ir
}

Received 2015 May 27; Accepted 2015 November 29.

\begin{abstract}
Context: Obesity has now become a common health problem worldwide. To gain insight into the epidemiology of the problem in Iran, we systematically reviewed all available studies on the prevalence of overweight and obesity in the Iranian pediatric population.

Evidence Acquisition: We systematically searched PubMed, ISI, SCOPUS, as well as Iranmedex, Irandoc, and Scientific Information Database (SID) databases.All studies on mean and standard deviation or percentile categories of BMI, WC, WHR, or WHtR or prevalence of obesity/overweight in Iranian child and adolescence, were performed from January 1990 to the end of December 2013. Refining processes were conducted by two independent reviewers. Quality assessment and data extraction followed based on validated form. As, these data were heterogeneous, meta-analysis was not performed.

Results: From 3253 records, through three refining steps, 129 articles were found related to our study. In Iran, national studies are limited and nearly there is no comprehensive study for sub-national trends. Different age and sex groups had large variations in the prevalence of obesity and overweight (from $1 \%$ up to $16.1 \%$ and from $4.4 \%$ up to $42.3 \%$ respectively for obesity and overweight).

Conclusions: Related data are very scattered or limited to some specific subgroups in some living areas. For comparing, aggregating, and imputing the information we need more modern practical statistical methods.
\end{abstract}

Keywords: Obesity, Overweight, Pediatrics, Systematic Review

\section{Context}

The global burden of disease (GBD) studies in 1990, 2000, and 2013 showed that metabolic risk factors (MRFs) are the most important determinants of emerging noncommunicable diseases all over the world (1-8). Obesity has now become a common health problem and its prevalence continues to increase in both developed and developing countries (9-11). The increasing incidence of childhood obesity and its attributed socioeconomic and public health burden is a real threat for developing countries (12). Recent studies reveal the increasing rates of overweight and obesity and their attributed ranges of adverse health outcomes in children and adolescents $(13,14)$. Most obese children and adolescents already are at high risk for metabolic complications, and for a wide range of morbidities $(15,16)$. Moreover, there are some evidence on longterm premature mortality and physical morbidity in their adulthood $(16,17)$.

Despite priority of the problem, there is an evident gap in the related literature on these topics $(11,18)$. Even though there are a few studies on trend and point estimations of BMI trend and prevalence of obesity in Iranian pediatric population, there are little information about their exposure distribution at sub-national level and no information about their trends and their effects on the health $(7,12,19)$. Most of available reports are scattered or limited to specific sub groups of population $(9,12)$.

Remarkably, the reported basal information on prevalence of overweight and obesity varies considerably from one study to another. They are recruited based on different measures from different target groups of different scopes with quite different age and sex distributions $(9,18)$, so that there is a growing need to prepare primary data to bridge health research to policy recommendations $(18,20)$. To address this issue, we need to provide comprehensive scientific evidence for triggering policy actions, controlling the programs, and measuring the effect of interventions (21).

Considering these, the main objective of our study was to systematically review of all available studies on the means and standard deviations of anthropometric mea- 
sures including body mass index (BMI); waist circumference (WC); waist-hip ratio (WHR); and waist-to-height ratio (WHtR), or reported prevalence of obesity or overweight by sex, age, and year at national and sub-national levels from 1990 to 2013 in Iranian children and adolescents.

\section{Evidence Acquisition}

The design and protocol of the study have been described in more detail earlier $(22,23)$. Here we refer to some essential points in brief.

\subsection{Outcomes Definition}

The world health organization (WHO), U.S. centers for disease control and prevention, and international obesity task force each have presented different definitions of overweight and obesity in children and adolescents (24-27) (Table 1).

\subsection{Measures Definition}

We have included studies that reported mean and standard deviation or percentile categories of BMI, WC, WHR, or WHtR or prevalence of obesity or overweight by sex, age, and year at national and sub-national levels in Iran, based on anthropometric measures. For each measure, the standardized protocols, definition and cut off were considered.

\subsection{Search Strategy}

To assess papers on obesity and/or overweight of Iranian children and adolescents, we searched PubMed and NLM Gateway (for MEDLINE), Institute of Scientific Information (ISI), and SCOPUS as the main international electronic data sources. Moreover Iranmedex, Irandoc, and scientific information database (SID), considered to the main domestic databases that have systematic search capability and the most coverage of national indexed or even non indexed Iranian scientific journals (Table 2). All Iranian scientific journals of medical universities that are not listed in the domestic electronic databases, governmental reports, projects reports, conferences and reference lists, were reviewed by hand searching. The Endnote version 11 reference manager software was used to manage the data.

We limited the search to national, provincial, district, community population based studies in Iranian children and adolescents (ages 6 - 18 years) and there was no restriction on language. Databases were searched from January 1990 to the end of December 2013.

\subsection{National Data Source}

We used the aggregated data of childhood and adolescence surveillance and prevention of adult noncommunicable disease (CASPIAN) study as the main available national data source for the cardio metabolic risk factors of children and adolescents in Iran (28). Data were collected in four different surveys during 2003 to 2012 at the national and sub-national levels in Iran (20, 29-34).We assessed first, third, and fourth rounds data since second rounds of data were not available.

\subsection{Study Selection and Eligibility Criteria}

We excluded papers on non-population-based studies, or those with duplicate citations. The studies that focused on specific populations (such as school-based studies) were excluded. When there were multiple publications on the same population, only the largest study or the main source of data was included. We used GBD (global burden of diseases) validated quality assessment. Papers that had poor ratings were excluded and data were extracted from moderate and high quality studies. The quality assessment has been followed independently by two research experts and probable discrepancy between them resolved based on third expert opinion. Agreement was assessed using Cohen's kappa statistic. The kappa statistic for agreement on quality assessment was 0.92 .

\subsection{Data Extraction}

The data extraction sheet contained the following items: general information of study and its citation; population detailed characteristics; methodological information of designing and conduction of study (the study region, scope of study (local study or survey), total sample size, age and sex groups, urban/rural areas, cut-off point of prevalence, reported prevalence and its 95\% confidence interval), and study outcomes indicators (age specific prevalence of overweight and/or obesity; body mass index mean; waist circumference mean; waist to hip ratio mean).

\section{Results}

We refined data for prevalence of obesity and overweight by sex, age, province, and year $(n=22972)$. Based on our search strategy we found 3253 records; of these 1875 were from international data bases and the remaining 1378 were obtained from national data bases. After removing duplicates, via the refining steps, only 129 articles were found related to our study domain. Figure 1 is a flowchart for the data collection and selection process.

Considering the inclusion and exclusion criteria, 62 articles that met our eligibility criteria were selected. From 
Table 1. Definitions of Overweight and Obesity in Children and Adolescents

\begin{tabular}{|c|c|}
\hline Organization & Definition \\
\hline World health organization & $\begin{array}{l}\text { WHO child growth standards (birth to age 5); obesity: body mass index }(\mathrm{BMI})>3 \text { standard deviations above the } \\
\text { WHO growth standard median; overweight: BMI }>2 \text { standard deviations above the WHO growth standard } \\
\text { median; underweight: BMI < } 2 \text { standard deviations below the WHO growth standard median; WHO reference } \\
2007 \text { (ages } 5 \text { to } 19) \text {; obesity: body mass index }(\mathrm{BMI})>2 \text { standard deviations above the WHO growth standard } \\
\text { median; overweight: BMI > } 1 \text { standard deviation above the WHO growth standard median; underweight: BMI }<2 \\
\text { standard deviations below the WHO growth standard median. }\end{array}$ \\
\hline U.S. centers for disease control and prevention & $\begin{array}{l}\text { In children ages } 2 \text { to } 19, \text { BMI is assessed by age- and sex-specific percentiles; obesity: BMI } 95 \text { th percentile } \leq \text {; } \\
\text { Overweight: BMI } 85 \text { th }<\text { and } \leq 95 \text { th percentile; normal weight: BMI } 5 \text { th }<\text { and } \leq 85 \text { th percentile; underweight: } \\
\text { BMI }<5 \text { th percentile. }\end{array}$ \\
\hline International obesity task force & $\begin{array}{l}\text { Provides international BMI cut points by age and sex for overweight and obesity for children age } 2 \text { to } 18 \text {. The cut } \\
\text { points correspond to an adult BMI of } 25 \text { (overweight) or } 30 \text { (obesity). }\end{array}$ \\
\hline
\end{tabular}

Table 2. The Search Strategy

\begin{tabular}{|c|c|}
\hline Search Strategy & High Body Mass Index (BMI)/Waist Circumference/Waist-Hip Ratio Waist-to-Height Ratio \\
\hline Search strategy in PubMed/Medline & 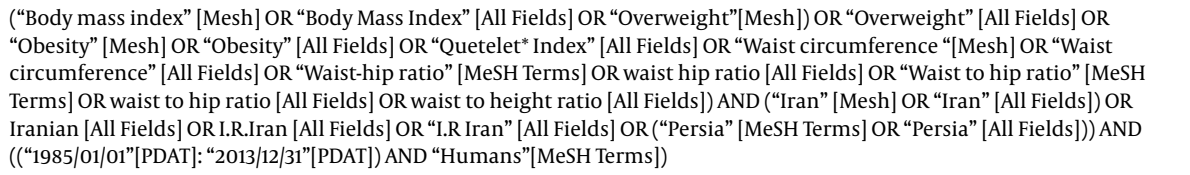 \\
\hline Search strategy in ISI Web of Science & $\begin{array}{l}\text { Time span = } 1990 \text { - 2013. Databases = SCI-EXPANDED, SSCI, CPCI-S, CPCI-SSH.Topic = ("Body Mass Index" OR "Overweight" OR } \\
\text { "Obesity" OR "Quetelet* Index" OR "Waist Circumference" OR "Waist hip ratio" OR "Waist to hip ratio" OR "Waist-hip ratio" OR } \\
\text { "Waist to height ratio") AND ("Iran" OR Iranian OR I.R.Iran OR "persia") OR Address= (Iran)) }\end{array}$ \\
\hline Search strategy in Scopus & $\begin{array}{l}\text { (TITLE-ABS-KEY (“Body Mass Index" OR “Overweight” OR “Obesity” OR “Quetelet* Index” OR “Waist Circumference “ OR “Waist } \\
\text { hip ratio “ OR “Waist to hip ratio" OR “Waist-hip ratio” OR “Waist to height ratio" OR “Waist-to- height ratio”)) AND } \\
\text { (TITLE-ABS-KEY (Iran OR Iranian OR I.R.Iran OR Persia) OR (AFFIL (Iran)) AND PUBYEAR > 1989 AND PUBYEAR < } 2013\end{array}$ \\
\hline IranMedex, SID and Irandoc & $\begin{array}{l}\text { "Body Mass Index", “BMI”, “Overweight”, “Obesity”, “Quetelet* Index”, “Waist-hip ratio”, “Waist to ratio”, “Chaghi”, } \\
\text { "Shakhesetudeh e badani”, “Ezafevazn”, “Dore kamar”, "Dore kamar be lagan”, "Dore kamar be basan”, “Dore kamar be ghad”, } \\
\text { in combination with terms pediatr* OR child* OR adolescent OR student OR teenager OR boys OR girls koodak, atfal, } \\
\text { nowjavan, daneshamooz, madreseh, madares, dokhtar, pesar in Persian language search. }\end{array}$ \\
\hline
\end{tabular}

these, the extracted results of 53 papers are reported in Tables 3 - 10 and the others are shown with related information in Tables 11 - 13.

Tables 3 - 10 shows the prevalence of obesity based on BMI, in eligible population-based studies in Iranian children and adolescents. Also for more precise comparison, the confidence interval for 95\% significance level (CI 95\%) was calculated for possible cases. We have also included each study designed.

Considering the systematic review results; the number of total population and points of data were 22972 and 29, 38985 and 47 respectively for boys and girls. There were 5 studies that did not report BMI separately for boys and girls. As well as regarding the geographically distribution we found, 9 national, 14 provincial, and 58 district level points of data.

The findings are scattered, with very wide ranges of values for BMI and for rates of obesity and overweight. On the other hand, non-standard classifications of age groups led to greater complexity in estimation of values. For in- stance, information about elementary school students was provided with at least 6 different age categories including: 6 - 10, 6 - 11, 6 - 12, 6 -14, 7 -11, and 7 -12. The lowest rate of obesity in this age group was 3.5\% which was reported in Yazd and Sabzevar $(9,38,50)$ and the highest rate of $17.7 \%$ was in Ahvaz (42). Similarly there were different reports for 10 - 15 year olds; based on one of them $13 \%$ of boys and $6.5 \%$ of girls in Tehran province were obese (41), in another study via the refining steps, only 129 articles were found related to our study domain in the same province reported 7.5\% and 7.3\% respectively for boys and girls (55). In 15 - 19 year old group, the prevalence of obesity was $2.8 \%$ for boys and $1.7 \%$ for girls $(49,57)$.

In Tables 11 - 13, the WHR, WC, and WHtR mean in population-based studies in Iranian children and adolescents reported based on papers data availability.

Tables 11 - 13 include only 9 papers with information on WHR, WC, or WHtR that met the study eligible criteria. The aggregated data CASPIAN studies are presented as the main source of national data. 


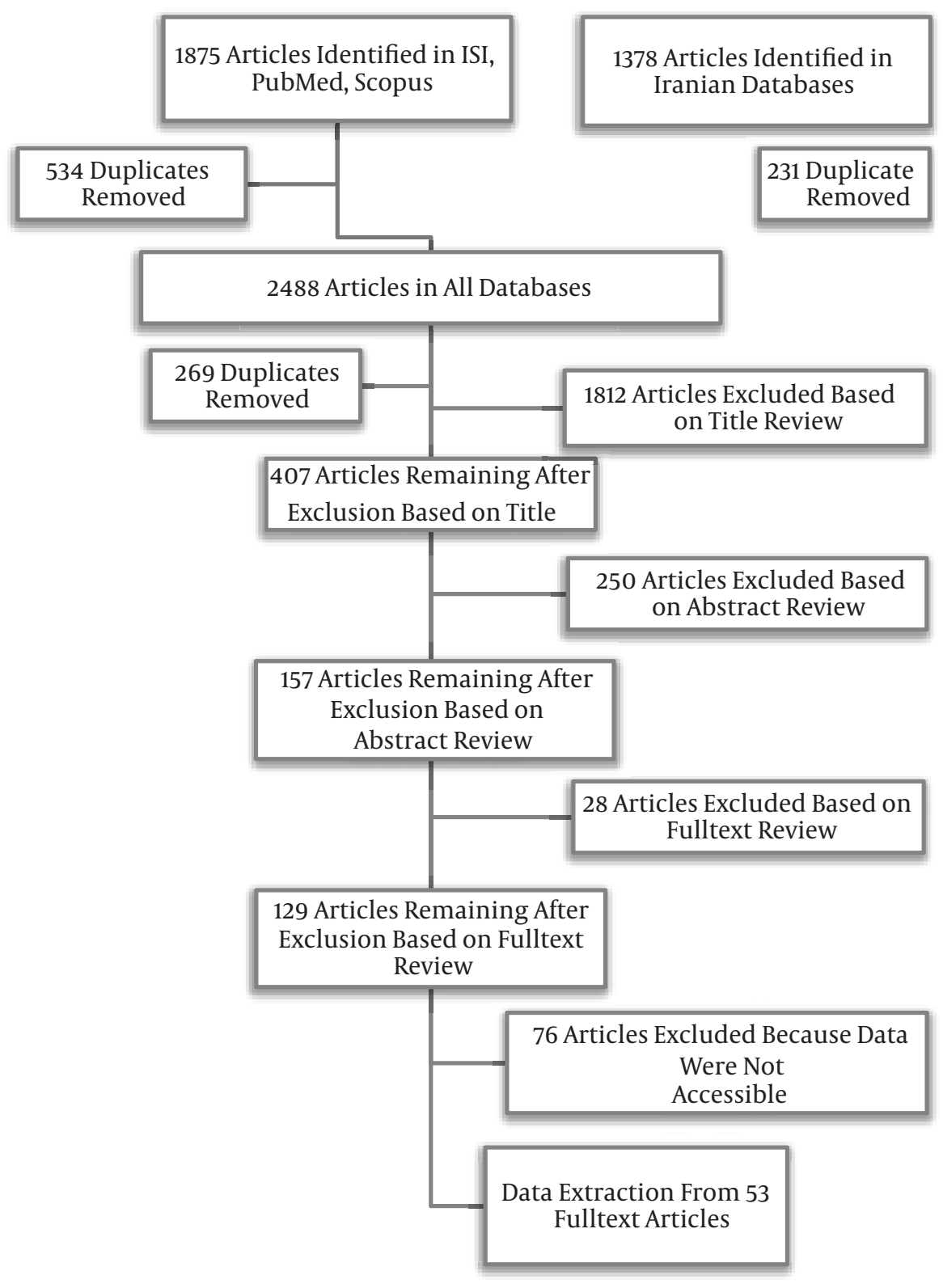

Figure 1. Flowchart for the Data Collection and Selection Process

Based on the first CASPIAN study in 2004, the national prevalence of abdominal obesity was $9.27(8.87 \%-9.67 \%)$ for both sexes, $9.14(8.60 \%$ - 9.69\%) for males, and $9.41(8.84 \%$ - 9.99\%) for females. In the last estimation for 2012 these were increased respectively to $19.12(18.22 \%-20.02 \%), 20.41$ (19.09\% - 23.05\%), and 17.79 (16.56\% - 19.02\%). In similar time period, the national mean of WC from $64.61(64.46 \mathrm{~cm}$ $64.76 \mathrm{~cm})$ rose to $67.02(66.57 \mathrm{~cm}-67.48 \mathrm{~cm})$.

\section{Discussion}

This review of our finding, similar to some regional and global studies, provides alarming evidence-based data on the considerable prevalence of childhood and adolescents overweight $(9,12,16,17)$. In Iran national studies, especially in pediatric groups, are limited and nearly there is no comprehensive study for sub-national trends. Considering the results of CASPIAN, as the only valid national study; in 2004, the national prevalence of obesity for $6-18$ 


\begin{tabular}{|c|c|c|c|c|c|c|c|c|c|c|c|c|c|}
\hline Reference & Location & Urban/Rural & $\begin{array}{l}\text { Year of } \\
\text { Study }\end{array}$ & Gender & $\begin{array}{l}\text { Age- } \\
\text { group, } \\
\mathbf{y}\end{array}$ & $\mathrm{Age}^{\mathrm{a}}$ & $\begin{array}{l}\text { Sample } \\
\text { Size, } \mathbf{n}\end{array}$ & BMI $^{\mathbf{a}}$ & $\begin{array}{c}\text { CI 95\% } \\
\text { BMI Mean }\end{array}$ & $\begin{array}{c}\text { Obesity } \\
\text { Definition }\end{array}$ & $\begin{array}{c}\text { Prevalence } \\
\text { of } \\
\text { Obesity/CI } \\
95 \%\end{array}$ & $\begin{array}{c}\text { Over } \\
\text { Weight } \\
\text { Definition }\end{array}$ & $\begin{array}{c}\text { Prevalence } \\
\text { of Over } \\
\text { Weight/CI } \\
95 \%\end{array}$ \\
\hline \multirow{3}{*}{$\begin{array}{l}\text { Aazami et } \\
\text { al. (2012) } \\
(35)\end{array}$} & $\begin{array}{l}\text { Kermanshah, } \\
\text { local study }\end{array}$ & $\mathrm{U}$ & 2010 & & & & & & & CDC 2000 & & CDC 2000 & \\
\hline & & & & Male & $\mathrm{NA}$ & $9.3 \pm 1.5$ & 756 & $17.44 \pm 3.2$ & $17.21-17.67$ & & $\begin{array}{c}13.4(12.57- \\
14.23)\end{array}$ & & $\begin{array}{c}12.6(11.81- \\
13.39)\end{array}$ \\
\hline & & & & Female & $\mathrm{NA}$ & $9.3 \pm 1.4$ & 644 & $17.11 \pm 3.1$ & $16.87-17.35$ & & $\begin{array}{c}8.1(7.53- \\
8.67)\end{array}$ & & $\begin{array}{c}14.3(13.35- \\
15.25)\end{array}$ \\
\hline \multirow[t]{2}{*}{$\begin{array}{l}\text { Abdollahi } \\
\text { et al. } \\
\text { (2010)(36) }\end{array}$} & $\begin{array}{l}\text { Golestan, } \\
\text { provencial } \\
\text { study }\end{array}$ & $\mathrm{U}$ & 2005 & & & & & & & wHO & & wHO & \\
\hline & & & & Female & & $\begin{array}{l}43.4 \pm \\
13.58\end{array}$ & 2500 & $\begin{array}{c}27.63 \pm \\
5.54\end{array}$ & $27.41-27.85$ & & $\begin{array}{c}30.7(29.87- \\
31.53)\end{array}$ & & $\begin{array}{c}35(34.11- \\
35.8)\end{array}$ \\
\hline \multirow{4}{*}{$\begin{array}{l}\text { Ahmadi et } \\
\text { al. (2010) } \\
\text { (37) }\end{array}$} & $\begin{array}{l}\text { Kerman, } \\
\text { local study }\end{array}$ & $\mathrm{U}$ & 2009 & & & & & & & $\begin{array}{c}>2 \mathrm{SD} \text { of } \\
\text { mean }\end{array}$ & & $\begin{array}{c}>2 \mathrm{SD} \text { of } \\
\text { mean }\end{array}$ & \\
\hline & & & & Both & $7-11$ & $9 \pm 1.4$ & 1566 & $16 \pm 3$ & $15.85-16.15$ & & $\begin{array}{c}9.7(9.27- \\
10.13)\end{array}$ & & $\begin{array}{c}4.4(4.19- \\
4.61)\end{array}$ \\
\hline & & & & Male & & $\mathrm{NA}$ & 770 & $16 \pm 2.9$ & $15.79-16.20$ & & $\mathrm{NA}$ & & $\mathrm{NA}$ \\
\hline & & & & Female & & $\mathrm{NA}$ & 796 & $16.1 \pm 3.1$ & $15.88-16.32$ & & $\mathrm{NA}$ & & $\mathrm{NA}$ \\
\hline \multirow{4}{*}{$\begin{array}{l}\text { Akhavan- } \\
\text { Karbasi et } \\
\text { al. (2009) } \\
\text { (38) }\end{array}$} & $\begin{array}{l}\text { Yazd, local } \\
\text { study }\end{array}$ & $\mathrm{U}$ & 2006 & & & & & & & $\mathrm{CDC} 2000$ & & CDC 2000 & \\
\hline & & & & Both & $6-6.9$ & $\mathrm{NA}$ & 400 & $\mathrm{NA}$ & $\mathrm{NA}$ & & $\begin{array}{c}3.8(3.29- \\
4.31)\end{array}$ & & $\begin{array}{c}4.3(3.73- \\
4.78)\end{array}$ \\
\hline & & & & Male & & $\mathrm{NA}$ & 200 & $\mathrm{NA}$ & $\mathrm{NA}$ & & $\begin{array}{c}5.5(4.78- \\
6.22)\end{array}$ & & $\begin{array}{l}5(4.34- \\
5.66)\end{array}$ \\
\hline & & & & Female & & $\mathrm{NA}$ & 200 & $\mathrm{NA}$ & $\mathrm{NA}$ & & $\begin{array}{l}2(1.73- \\
2.27)\end{array}$ & & $\begin{array}{c}3.5(3.03- \\
3.97)\end{array}$ \\
\hline \multirow{4}{*}{$\begin{array}{l}\text { Mirzazadeh } \\
\text { et al. } \\
(2000)(9)\end{array}$} & $\begin{array}{l}\text { Tehran, } \\
\text { local study }\end{array}$ & $\mathrm{u}$ & 2002 & & & & & & & CDC 2000 & & CDC 2000 & \\
\hline & & & & Both & $10 \cdot 19$ & $\mathrm{NA}$ & 421 & $\mathrm{NA}$ & $\mathrm{NA}$ & & $\begin{array}{c}3.8(3.45- \\
4.15)\end{array}$ & & $\mathrm{NA}$ \\
\hline & & & & Male & & & 177 & $\mathrm{NA}$ & $\mathrm{NA}$ & & $\begin{array}{c}5.1-(4.39- \\
5.81)\end{array}$ & & $\mathrm{NA}$ \\
\hline & & & & Female & & & 244 & $\mathrm{NA}$ & $\mathrm{NA}$ & & $\begin{array}{c}2.8(2.46- \\
3.14)\end{array}$ & & $\mathrm{NA}$ \\
\hline
\end{tabular}

Abbreviation: CI, confidence interval; $\mathrm{F}$, female; $\mathrm{M}$, male; $\mathrm{NA}$, not available; $\mathrm{R}$, rural; $\mathrm{R}$, rural total; $\mathrm{T}$, total; $\mathrm{U}$, urban; $\mathrm{UT}$, urban total; $\mathrm{y}$, year. Values are expressed as mean (SD).

year olds male and female was respectively 3.34 (3\% - 3.69\%) and 3.50 (3.15\% - 3.87\%). In 2010 these estimations respectively rose to $13.58(12.59 \%-14.63 \%)$, and $7.68(6.71 \%-8.72 \%)$. In the last estimation for 2012 these were increased to; 13.58 (12.59\% - 14.63\%), and 10.15 (9.27\% - 11.09\%).

Different age and sex groups had large variations in the prevalence of obesity and overweight. As a considerable point; there are also a lot of missing data for different sub groups which is leading from the misclassification or other limitation of research papers' data presentation $(89,90)$. Some of these diversities are attributed to the geographical scopes of studies; some estimations belong to local level studies, some others estimate district level and others are designed for national estimations. Moreover, the quality of presented data, and some methodological problems, in designing and conducting the related researches, were other sources of diversity $(30,32,85)$. There are also some visible data lags in some areas of country or for different target groups that should be more considered for future studies' plan. For all measures however the reports have significant missing in reporting the confidence intervals for both measures values' and obesity/overweight rates, which limited our ability to compare and analyze results. On the other hand, most of our efforts to contact study authors for requesting more data and information failed.

In Iran there are few national studies and nearly no comprehensive study for sub-national trends (91-93). In comparison with other related studies, compared with the 


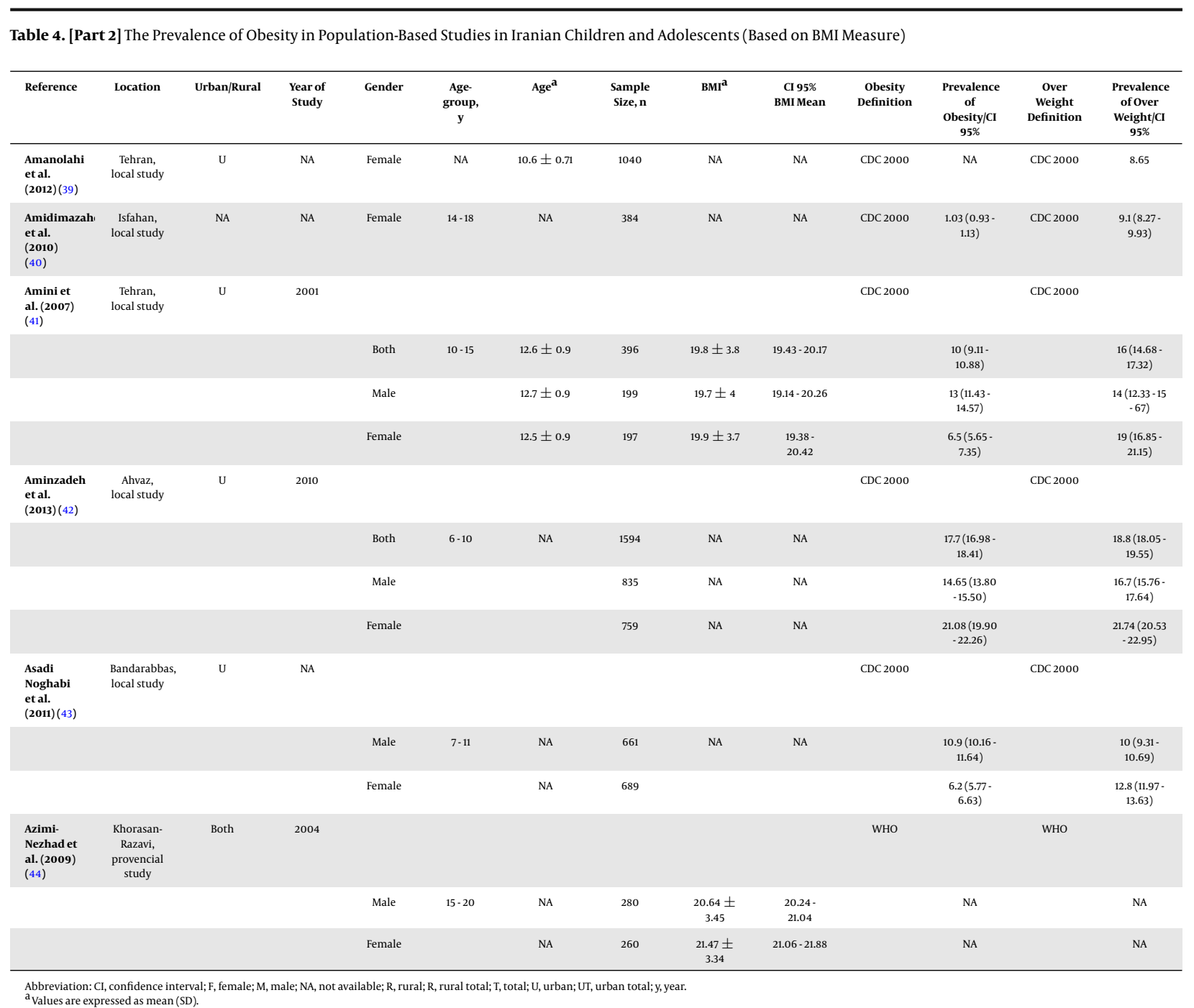

GBD studies with overall 102 points of data, we benefit from more data driven estimations rather than the model driven results. We will provide more data points that lead to higher quality of estimation $(1,4-8,19)$. This is the first comprehensive systematic approach to search and data gathering, during which we benefited from the highest level of access to the published, available unpublished and grey literature through the comprehensive hand searching process. We had access to main national sources of CASPIAN study $(30,32,85)$.

Considering previous studies, the present study has several achievements. This study presents the most scientific evidences for the prevalence and trends of obesity and overweight in pediatric population from 1990 to 2013. We benefited from all available sources of data alongside the advanced comprehensive search strategies. All of do- mestic data bases were searched exactly with all of English/Persian equivalent search terms.

Because of scarcity of data, variations in groups studied, differences in living areas (urban/rural), discrepancy of the measures, for comparing and aggregating the information, we need more modern practical statistical methods $(9,12)$. These methods are advanced regression models, using existing data, benefit from models for age, hierarchical pattern of data, spatial and temporal pattern of data, and covariates $(94,95)$.

The present study summarized the information of studies on mean and standard deviation of different measures including BMI, WC, WHR, or WHtR and reported prevalence of obesity or overweight by sex, age, and year at national and sub-national levels from 1990 to 2013 in Iranian children and adolescents. 
Table 5. [Part 3] The Prevalence of Obesity in Population-Based Studies in Iranian Children and Adolescents (Based on BMI Measure)

\begin{tabular}{|c|c|c|c|c|c|c|c|c|c|c|c|c|c|}
\hline Reference & Location & Urban/Rural & $\begin{array}{l}\text { Year of } \\
\text { Study }\end{array}$ & Gender & $\begin{array}{l}\text { Age- } \\
\text { group, } \\
\mathbf{y}\end{array}$ & $\mathrm{Age}^{\mathrm{a}}$ & $\begin{array}{c}\text { Sample } \\
\text { Size, } \mathbf{n}\end{array}$ & BMI $^{\mathbf{a}}$ & $\begin{array}{c}\text { CI 95\% } \\
\text { BMI Mean }\end{array}$ & $\begin{array}{c}\text { Obesity } \\
\text { Definition }\end{array}$ & $\begin{array}{c}\text { Prevalence } \\
\text { of } \\
\text { Obesity/CI } \\
95 \%\end{array}$ & $\begin{array}{c}\text { Over } \\
\text { Weight } \\
\text { Definition }\end{array}$ & $\begin{array}{c}\text { Prevalence } \\
\text { of Over } \\
\text { Weight/CI } \\
95 \%\end{array}$ \\
\hline $\begin{array}{l}\text { Bazhan et } \\
\text { al. (2005) } \\
\text { (45) }\end{array}$ & $\begin{array}{l}\text { Lahijan, } \\
\text { local study }\end{array}$ & $\mathrm{u}$ & 2001 & Female & $14-17$ & $\mathrm{NA}$ & 400 & $21.9 \pm 3.5$ & $21.56-22.24$ & CDC 2000 & $\begin{array}{l}5.3(4.81- \\
5.79)\end{array}$ & CDC 2000 & $\begin{array}{c}14.8(13.56- \\
16.04)\end{array}$ \\
\hline $\begin{array}{l}\text { Bidad et } \\
\text { al.(2008) } \\
(46)\end{array}$ & $\begin{array}{l}\text { Tehran, } \\
\text { local study }\end{array}$ & $\mathrm{u}$ & 2005 & Female & $11-17$ & $14 \pm 1.8$ & 400 & $\begin{array}{c}20.61 \pm \\
3.79\end{array}$ & $\begin{array}{l}20.24- \\
20.98\end{array}$ & CDC 2000 & $\begin{array}{c}6.7(6.09- \\
7.31)\end{array}$ & $\mathrm{CDC} 2000$ & $\begin{array}{c}14.6(13.38 \text { - } \\
15.82)\end{array}$ \\
\hline \multirow{4}{*}{$\begin{array}{l}\text { Baygi et } \\
\text { al.(2010) } \\
(47)\end{array}$} & $\begin{array}{l}\text { Neishabour, } \\
\text { local study }\end{array}$ & $\mathrm{u}$ & 2005 & & & & & & & CDC 2000 & & CDC 2000 & \\
\hline & & & & Both & $6-12$ & $\mathrm{NA}$ & 1471 & $\mathrm{NA}$ & $\mathrm{NA}$ & & $\begin{array}{c}4.6(4.38- \\
4.82)\end{array}$ & & $\mathrm{NA}$ \\
\hline & & & & Male & & $\mathrm{NA}$ & 822 & $\mathrm{NA}$ & $\mathrm{NA}$ & & $\mathrm{NA}$ & & $\mathrm{NA}$ \\
\hline & & & & Female & & $\mathrm{NA}$ & 649 & $\mathrm{NA}$ & $\mathrm{NA}$ & & $\mathrm{NA}$ & & $\mathrm{NA}$ \\
\hline $\begin{array}{l}\text { Gargari et } \\
\text { al. (2004) } \\
(48)\end{array}$ & $\begin{array}{c}\text { Tabriz, } \\
\text { local study }\end{array}$ & $\mathrm{u}$ & 2001 & Female & $14-17.9$ & $16 \pm 1.4$ & 1518 & $21.3 \pm 3.6$ & $21.12-21.48$ & CDC 2000 & $\mathrm{NA}$ & CDC 2000 & $\mathrm{NA}$ \\
\hline \multirow{3}{*}{$\begin{array}{l}\text { Gharakhanlo } \\
\text { et al. } \\
\text { (2012) (49) }\end{array}$} & $\begin{array}{l}\text { National } \\
\text { study }\end{array}$ & u & 2011 & & & & & & & шно & & wHO & \\
\hline & & & & Male & $15-19$ & $\mathrm{NA}$ & 139 & $3.1 \pm 21.4$ & $20.88-21.92$ & & $\begin{array}{c}2.8(2.35- \\
3.25)\end{array}$ & & $\begin{array}{c}9.3(7.90- \\
10.70)\end{array}$ \\
\hline & & & & Female & & $\mathrm{NA}$ & 145 & $21.9 \pm 3.8$ & $21.28-22.52$ & & $\begin{array}{c}2.7(2.27- \\
3.13)\end{array}$ & & $\begin{array}{c}12.4(10.63- \\
14.17)\end{array}$ \\
\hline \multirow{3}{*}{$\begin{array}{l}\text { Haeri Be- } \\
\text { hbahani } \\
\text { et al. } \\
(2009) \\
(50)\end{array}$} & $\begin{array}{l}\text { Sabzevar, } \\
\text { local study }\end{array}$ & u & 2007 & & & & & & & CDC 2000 & & CDC 2000 & \\
\hline & & & & Both & $6-11$ & $\mathrm{NA}$ & 960 & $16.6 \pm 2.8$ & $16.42-16.78$ & & $6(4.5-7.6)$ & & $\begin{array}{c}8.4(6.7- \\
10.2)\end{array}$ \\
\hline & & & & & & $\mathrm{NA}$ & 840 & $16.3 \pm 2.6$ & $16.12-16.48$ & & $\begin{array}{c}3.5(2.2- \\
4.7)\end{array}$ & & $\begin{array}{l}7.4(5.6- \\
9.2)\end{array}$ \\
\hline $\begin{array}{l}\text { Hajian et } \\
\text { al.(2008) } \\
(51)\end{array}$ & $\begin{array}{l}\text { Babol, local } \\
\text { study }\end{array}$ & $\mathrm{u}$ & 2006 & Both & $7-12$ & $\mathrm{NA}$ & 1000 & $\mathrm{NA}$ & & CDC 2000 & $\begin{array}{c}5.8(5.46- \\
6.14)\end{array}$ & $\mathrm{CDC} 2000$ & $\begin{array}{c}12.3(11.63- \\
12.97)\end{array}$ \\
\hline \multirow{4}{*}{$\begin{array}{l}\text { Hajian- } \\
\text { Tilaki et } \\
\text { al.(2011) } \\
(52)\end{array}$} & $\begin{array}{l}\text { Babol, local } \\
\text { study }\end{array}$ & $\mathrm{u}$ & 2006 & & & & & & & CDC 2000 & & CDC 2000 & \\
\hline & & & & Both & $7 \cdot 12$ & $9.3 \pm 1.5$ & 1000 & $\mathrm{NA}$ & $\mathrm{NA}$ & & $\begin{array}{l}5.8(5.46- \\
6.14) \mathrm{F}: 3.8\end{array}$ & & $\begin{array}{l}\text { 12.3(11.63- } \\
\text { 12.96)M: } \\
\text { 12.5F: }\end{array}$ \\
\hline & & & & Male & & $\mathrm{NA}$ & 450 & $16.8 \pm 3.4$ & $16.49-17.14$ & & $\begin{array}{c}8.8(8.06- \\
9.54)(7-9 \\
\text { yo: } 8.77)(10 \\
-12 \text { yo: } \\
10.43)\end{array}$ & & $\begin{array}{c}12.5(11.49- \\
13.51)(7-9 \\
\text { yo: } 12.23) \\
(10-12 \text { yo: } \\
11.15)\end{array}$ \\
\hline & & & & Female & & $\mathrm{NA}$ & 550 & $16.7 \pm 2.8$ & $16.47-16.93$ & & $\begin{array}{c}3.8(3.46- \\
4.11)(7-9 \\
\text { yo:5.02)(10 } \\
-12 \text { yo: } \\
2.99)\end{array}$ & & $\begin{array}{c}12.2(11.30- \\
13.09)(7-9 \\
\text { yo: } 9.9)(10- \\
12 \text { yo: } 16.54)\end{array}$ \\
\hline
\end{tabular}

Abbreviation: $\mathrm{Cl}$, confidence interval; $\mathrm{F}$, female; $\mathrm{M}$, male; $\mathrm{NA}$, not available; $\mathrm{R}$, rural; $\mathrm{R}$, rural total; $\mathrm{T}$, total; $\mathrm{U}$, urban; $\mathrm{UT}$, urban total; $\mathrm{y}$, year. ${ }^{\mathrm{a}}$ Values are expressed as mean (SD).

Obesity seems to be one of the major public health problems. Considering that, several methods have been proposed in the field of medical and surgical treatments. In this context, recent promising approaches emphasize on the population based interventions impacts (96). Aiming that, we call for a sustained valid data sources to monitor, prevent, and control of pediatric overweight and obesity. These processes should be followed through ongoing community-based lifestyle intervention on diet, physical activity promotion, and other local and national experi- ences $(31,56,87,97)$. Evidences reveal that for more effectiveness, these interventions should be started and exactly followed from the adolescence $(12,23,96)$.

Health researchers, professionals and policy-makers should focus on more evidence based policies which require more reliable data. Aiming that, all processes of designing; conducting; monitoring; and distribution of data should be managed based on advanced scientific methods $(18,98)$. 
Table 6. [Part 4] The Prevalence of Obesity in Population-Based Studies in Iranian Children and Adolescents (Based on BMI Measure)

\begin{tabular}{|c|c|c|c|c|c|c|c|c|c|c|c|c|c|}
\hline Reference & Location & Urban/Rural & $\begin{array}{l}\text { Year of } \\
\text { Study }\end{array}$ & Gender & $\begin{array}{l}\text { Age- } \\
\text { group, } \\
\mathbf{y}\end{array}$ & $\mathrm{Age}^{\mathrm{a}}$ & $\begin{array}{l}\text { Sample } \\
\text { Size, } \mathbf{n}\end{array}$ & BMI $^{\mathbf{a}}$ & $\begin{array}{c}\text { CI 95\% } \\
\text { BMI Mean }\end{array}$ & $\begin{array}{c}\text { Obesity } \\
\text { Definition }\end{array}$ & $\begin{array}{c}\text { Prevalence } \\
\text { of } \\
\text { Obesity/CI } \\
95 \%\end{array}$ & $\begin{array}{c}\text { Over } \\
\text { Weight } \\
\text { Definition }\end{array}$ & $\begin{array}{c}\text { Prevalence } \\
\text { of Over } \\
\text { Weight/CI } \\
95 \%\end{array}$ \\
\hline \multirow{4}{*}{$\begin{array}{l}\text { Hajian- } \\
\text { Tilaki et } \\
\text { al. (2012) } \\
(53)\end{array}$} & $\begin{array}{c}\text { Babol, } \\
\text { local study }\end{array}$ & u & $\mathrm{NA}$ & & & & & & & CDC 2000 & & CDC 2000 & \\
\hline & & & & Both & $12-17$ & $\mathrm{NA}$ & 1200 & $\mathrm{NA}$ & $\mathrm{NA}$ & & $\begin{array}{c}8.3(7.87- \\
8.73)\end{array}$ & & $\begin{array}{c}15.1(14.37 \\
15.82)\end{array}$ \\
\hline & & & & Male & & & $\mathrm{NA}$ & $\mathrm{NA}$ & & & 10.2 & & $\mathrm{NA}$ \\
\hline & & & & Female & & & $\mathrm{NA}$ & $\mathrm{NA}$ & & & 6.5 & & $\mathrm{NA}$ \\
\hline $\begin{array}{l}\text { Janghorbani } \\
\text { et al. } \\
(\mathbf{1 9 9 8 ) ( 5 4 )}\end{array}$ & $\begin{array}{l}\text { Kerman, } \\
\text { local study }\end{array}$ & U & 1995 & Female & $14-18$ & $16.2 \pm 1.3$ & 1000 & $19.8 \pm 2.9$ & $19.62-19.98$ & wHO & $\begin{array}{c}5.3(4.99- \\
5.61)\end{array}$ & WHO & $\begin{array}{c}21.9(20.84 \text { - } \\
22.96)\end{array}$ \\
\hline \multirow{4}{*}{$\begin{array}{l}\text { Karandish } \\
\text { et al. } \\
(2004) \\
(55)\end{array}$} & $\begin{array}{c}\text { Tehran, } \\
\text { local study }\end{array}$ & $\mathrm{u}$ & 2000 & & & & & & & CDC 2000 & & CDC 2000 & \\
\hline & & & & Both & $11-16$ & $\mathrm{NA}$ & 2486 & $\mathrm{NA}$ & $\mathrm{NA}$ & & $\mathrm{NA}$ & & $\mathrm{NA}$ \\
\hline & & & & Male & & $13.7 \pm 1.54$ & 1147 & $19.8 \pm 3.95$ & $\begin{array}{l}19.57- \\
20.03\end{array}$ & & $\begin{array}{c}7.3(6.91- \\
7.69)\end{array}$ & & $\begin{array}{c}23.3(22.27- \\
24.33)\end{array}$ \\
\hline & & & & Female & & $13.4 \pm 1.64$ & 1339 & $\begin{array}{c}20.63 \pm \\
4.11\end{array}$ & $\begin{array}{l}20.41- \\
20.85\end{array}$ & & $\begin{array}{c}8.3(7.89- \\
8.71)\end{array}$ & & $\begin{array}{c}18.8(17.98- \\
19.62)\end{array}$ \\
\hline \multirow{4}{*}{$\begin{array}{l}\text { Kelishadi } \\
\text { et al. } \\
\text { (2003) } \\
(56)\end{array}$} & $\begin{array}{c}\text { Isfahan, } \\
\text { Markazi } \\
\text { local study }\end{array}$ & Both & 2001 & & & & & & & CDC 2000 & & CDC 2000 & \\
\hline & & & & Both & $11-18$ & $\mathrm{NA}$ & 2000 & $\begin{array}{c}\mathrm{U}: 25.4 \pm \\
5.2 \mathrm{R}: 23.2 \\
\quad \pm 7.1\end{array}$ & $\begin{array}{c}\text { U: } 24.29- \\
26.51 \mathrm{R}: \\
22.76- \\
23.64\end{array}$ & & $\mathrm{NA}$ & & $\mathrm{NA}$ \\
\hline & & & & Male & & $\mathrm{NA}$ & 1000 & & & & $\begin{array}{c}1.87(1.79- \\
1.95)\end{array}$ & & $\begin{array}{c}7.4(7.10- \\
7.70)\end{array}$ \\
\hline & & & & Female & & $\mathrm{NA}$ & 1000 & & & & $\begin{array}{c}2.9(2.73- \\
3.07)\end{array}$ & & $\begin{array}{c}10.7(10.11- \\
11.29)\end{array}$ \\
\hline $\begin{array}{l}\text { Khadivzadeh } \\
\text { et al. } \\
(\mathbf{2 0 0 2})(57)\end{array}$ & $\begin{array}{c}\text { Mashad, } \\
\text { local study }\end{array}$ & U & 1998 & Female & $15-19$ & $\mathrm{NA}$ & 440 & $\mathrm{NA}$ & $\mathrm{NA}$ & CDC 2000 & $\begin{array}{c}1.8(1.63- \\
1.97)\end{array}$ & CDC 2000 & $\begin{array}{l}5.8(5.29- \\
6.31)\end{array}$ \\
\hline $\begin{array}{l}\text { Maddah } \\
(\mathbf{2 0 0 7 ) ( 5 8 )}\end{array}$ & $\begin{array}{l}\text { Guilan, } \\
\text { local study }\end{array}$ & u & 2005 & Female & $14-17$ & $\mathrm{NA}$ & 1054 & $\mathrm{NA}$ & $\mathrm{NA}$ & & $\begin{array}{c}\mathrm{F}: 5.2(4.90 \\
-5.50)\end{array}$ & & $\begin{array}{c}18.6(17.69- \\
19.51)\end{array}$ \\
\hline \multirow{4}{*}{$\begin{array}{l}\text { Maddah } \\
\text { et al. } \\
(\mathbf{2 0 1 0})(59)\end{array}$} & $\begin{array}{c}\text { Rasht, } \\
\text { local study }\end{array}$ & u & 2007 & & & & & & & CDC 2000 & & CDC 2000 & \\
\hline & & & & Both & $6-11$ & $\mathrm{NA}$ & 6635 & $\mathrm{NA}$ & $\mathrm{NA}$ & & $\mathrm{NA}$ & & $\mathrm{NA}$ \\
\hline & & & & Male & & & 3551 & & & & $\begin{array}{c}5(4.89 . \\
5.11)\end{array}$ & & $\begin{array}{c}11.5(11.26- \\
11.74)\end{array}$ \\
\hline & & & & Female & & & 3084 & & & & $\begin{array}{c}5.9(5.72- \\
6.08)\end{array}$ & & $\begin{array}{c}15(14.58- \\
15.42)\end{array}$ \\
\hline \multirow{4}{*}{$\begin{array}{l}\text { Maddah } \\
\text { et al. } \\
(2010) \\
(60)\end{array}$} & $\begin{array}{l}\text { Zahedan, } \\
\text { local study }\end{array}$ & u & $\mathrm{NA}$ & & & & & & & CDC 2000 & & CDC 2000 & \\
\hline & & & & Both & $7-11$ & $\mathrm{NA}$ & 1079 & $\mathrm{NA}$ & $\mathrm{NA}$ & & $\mathrm{NA}$ & & $\begin{array}{c}\text { M: } 8.9 \mathrm{~F}: \\
10.3\end{array}$ \\
\hline & & & & Male & & & 500 & & $\mathrm{NA}$ & & $\mathrm{NA}$ & & $\begin{array}{c}8.9(8.42- \\
9.38)\end{array}$ \\
\hline & & & & Female & & & 579 & $\mathrm{NA}$ & $\mathrm{NA}$ & & $\mathrm{NA}$ & & $\begin{array}{c}10.3(9.49 \text { - } \\
11.11)\end{array}$ \\
\hline
\end{tabular}

Abbreviation: $\mathrm{CI}$, confidence interval; $\mathrm{F}$, female; $\mathrm{M}$, male; $\mathrm{NA}$, not available; R, rural; R, rural total; $\mathrm{T}$, total; U, urban; $\mathrm{UT}$, urban total; $\mathrm{y}$, year.

\section{Conclusions}

In conclusion, the present findings could provide practical information on children and adolescents' overweight and obesity for better health policy and more detailed design studies in this area. Because of the importance of application of researches' data more policy attention should be considered for more efficient papers' data presentation. The presented results also could be used for future complementary sub-national, national or even global related studies. 
Table 7. [Part 5] The Prevalence of Obesity in Population-Based Studies in Iranian Children and Adolescents (Based on BMI Measure)

\begin{tabular}{|c|c|c|c|c|c|c|c|c|c|c|c|c|c|}
\hline Reference & Location & Urban/Rural & $\begin{array}{l}\text { Year of } \\
\text { Study }\end{array}$ & Gender & $\begin{array}{l}\text { Age- } \\
\text { group, } \\
\mathbf{y}\end{array}$ & Age $^{a}$ & $\begin{array}{c}\text { Sample } \\
\text { Size, } n\end{array}$ & BMI $^{\mathbf{a}}$ & $\begin{array}{c}\text { CI 95\% } \\
\text { BMI Mean }\end{array}$ & $\begin{array}{c}\text { Obesity } \\
\text { Definition }\end{array}$ & $\begin{array}{c}\text { Prevalence } \\
\text { of } \\
\text { Obesity/CI } \\
95 \%\end{array}$ & $\begin{array}{c}\text { Over } \\
\text { Weight } \\
\text { Definition }\end{array}$ & $\begin{array}{c}\text { Prevalence } \\
\text { of Over } \\
\text { Weight/CI } \\
95 \%\end{array}$ \\
\hline \multirow{4}{*}{$\begin{array}{l}\text { Mehrkash } \\
\text { et al. } \\
(2010)(61)\end{array}$} & $\begin{array}{c}\text { Gorgan, } \\
\text { local study }\end{array}$ & $\mathrm{u}$ & NA & & & & & & & CDC 2000 & & CDC 2000 & \\
\hline & & & & Both & $15-18$ & $\mathrm{NA}$ & 450 & $\mathrm{NA}$ & $\mathrm{NA}$ & & NA & & $\mathrm{NA}$ \\
\hline & & & & Male & & $\begin{array}{c}15.66 \pm \\
0.68\end{array}$ & 225 & $\begin{array}{c}21.24 \pm \\
3.45\end{array}$ & $\begin{array}{l}20.79- \\
21.69\end{array}$ & & $\mathrm{NA}$ & & $\mathrm{NA}$ \\
\hline & & & & Female & & $\begin{array}{c}16.34 \pm \\
0.77\end{array}$ & 225 & $\begin{array}{c}22.41 \pm \\
4.32\end{array}$ & $21.85-22.97$ & & $\mathrm{NA}$ & & $\mathrm{NA}$ \\
\hline $\begin{array}{l}\text { Mirhosseini } \\
\text { et al. } \\
\text { (2009) } \\
(62)\end{array}$ & $\begin{array}{l}\text { Mashhad, } \\
\text { local study }\end{array}$ & $\mathrm{u}$ & NA & Female & $15-17$ & $\begin{array}{c}16.4 \pm \\
0.09\end{array}$ & 622 & $20.7 \pm 3.6$ & $13.5-36.9$ & $\mathrm{NA}$ & $\mathrm{NA}$ & $\mathrm{NA}$ & $\mathrm{NA}$ \\
\hline \multirow{7}{*}{$\begin{array}{l}\text { Mirmiran } \\
\text { et al. } \\
(2004) \\
(63)\end{array}$} & $\begin{array}{c}\text { Tehran, } \\
\text { local study }\end{array}$ & U & & & & & & & & & & & \\
\hline & & & 1999 & Both & $6-16$ & $\mathrm{NA}$ & 732 & $\mathrm{NA}$ & $\mathrm{NA}$ & CDC 2000 & $\begin{array}{c}4.5(4.19- \\
4.81)\end{array}$ & CDC 2000 & $\mathrm{NA}$ \\
\hline & & & 1999 & Male & & & 339 & $\mathrm{NA}$ & & & $\begin{array}{c}5.5(4.95- \\
6.05)\end{array}$ & & $\mathrm{NA}$ \\
\hline & & & 1999 & Female & & & 393 & $\mathrm{NA}$ & & & $\begin{array}{c}3.7(3.35- \\
4.05)\end{array}$ & & $\mathrm{NA}$ \\
\hline & & & 2002 & Both & $6-16$ & $\mathrm{NA}$ & 673 & $\mathrm{NA}$ & $\mathrm{NA}$ & CDC 2000 & $\begin{array}{c}5.7(5.29 \text { - } \\
6.11)\end{array}$ & CDC 2000 & $\mathrm{NA}$ \\
\hline & & & 2002 & Male & & $\mathrm{NA}$ & 312 & & & & $\begin{array}{c}6.3(5.64- \\
6.96)\end{array}$ & & \\
\hline & & & 2002 & Female & & $\mathrm{NA}$ & 361 & & & & $\begin{array}{c}5.2(4.69 \text { - } \\
5.71)\end{array}$ & & \\
\hline \multirow{4}{*}{$\begin{array}{l}\text { Moayeri } \\
\text { et al. } \\
(2006) \\
(64)\end{array}$} & $\begin{array}{c}\text { Tehran, } \\
\text { local study }\end{array}$ & U & 2005 & & & & & & & CDC 2000 & & CDC2000 & \\
\hline & & & & Both & $11-18$ & $\mathrm{NA}$ & 2880 & $\mathrm{NA}$ & $\mathrm{NA}$ & & $\begin{array}{c}7.1(6.72- \\
7.48)\end{array}$ & & $\begin{array}{c}17.9(17.06- \\
18.74)\end{array}$ \\
\hline & & & & & & $\mathrm{NA}$ & 1180 & $\mathrm{NA}$ & $\mathrm{NA}$ & & $\begin{array}{c}7.8(7.54- \\
8.06)\end{array}$ & & $\begin{array}{c}21.1(20.49- \\
21.71)\end{array}$ \\
\hline & & & & & & $\mathrm{NA}$ & 1700 & $\mathrm{NA}$ & $\mathrm{NA}$ & & $\begin{array}{c}6.4(6.12- \\
6.68)\end{array}$ & & $\begin{array}{c}14.7(14.10- \\
15.30)\end{array}$ \\
\hline \multirow{3}{*}{$\begin{array}{l}\text { Mohammadpou } \\
\text { Ahranjani } \\
\text { et al. } \\
\text { (2003) } \\
\text { (65) }\end{array}$} & $\begin{array}{l}\text { ur-Tehran, } \\
\text { local study }\end{array}$ & $\mathrm{u}$ & 2001 & & & & & & & CDC 2000 & & CDC 2000 & \\
\hline & & & & Male & $11-16$ & $13.7 \pm 1.54$ & 1068 & $19.8 \pm 3.95$ & $\begin{array}{l}19.56- \\
20.04\end{array}$ & & $\begin{array}{c}7.3(6.89- \\
7.71)\end{array}$ & & $\begin{array}{c}18.8(17.88- \\
19.72)\end{array}$ \\
\hline & & & & Female & & $13.4 \pm 1.54$ & 1253 & $20.6 \pm 4.11$ & $\begin{array}{l}20.37- \\
20.83\end{array}$ & & $\begin{array}{c}8.3(7.88- \\
8.72)\end{array}$ & & $\begin{array}{c}23.1(22.12 \text { - } \\
24.08)\end{array}$ \\
\hline $\begin{array}{l}\text { Mojibian } \\
\text { et al. } \\
\text { (2001) } \\
(66)\end{array}$ & $\begin{array}{l}\text { Yazd, local } \\
\text { study }\end{array}$ & u & 2000 & Female & $15-65$ & $\mathrm{NA}$ & 570 & $\mathrm{NA}$ & $\mathrm{NA}$ & wно & $\begin{array}{c}16.3(15.18- \\
17.42)\end{array}$ & CDC 2000 & $\begin{array}{c}11.8(10.15- \\
13.45)\end{array}$ \\
\hline \multirow[t]{3}{*}{$\begin{array}{l}\text { Montazerifar } \\
\text { et al. } \\
(2009)(67)\end{array}$} & $\begin{array}{l}\text { Sistan va } \\
\text { Baluchis- } \\
\text { tan, local } \\
\text { study }\end{array}$ & $\mathrm{u}$ & 2006 & & & & & & & CDC 2000 & & CDC 2000 & \\
\hline & & & & Female & $14-18$ & $16.4 \pm 1.3$ & 752 & $20 \pm 1.45$ & $\mathrm{NA}$ & & $\begin{array}{l}1.5(1.39- \\
1.61)\end{array}$ & & $\begin{array}{c}\text { F: } 8.6(8.04 \\
-9.16)\end{array}$ \\
\hline & $\begin{array}{l}\text { Zahedan, } \\
\text { local study }\end{array}$ & & 2007 & Female & $11-14$ & $13 \pm 0.89$ & 687 & & & & $\begin{array}{c}1.7(1.58- \\
1.82)\end{array}$ & & $\begin{array}{c}8.7(8.11- \\
9.29)\end{array}$ \\
\hline
\end{tabular}

Abbreviation: CI, confidence interval; F, female; $\mathrm{M}$, male; NA, not available; R, rural; R, rural total; $\mathrm{T}$, total; U, urban; UT, urban total; $\mathrm{y}$, year.

Values are expressed as mean (SD) 
Table 8. [Part 6] The Prevalence of Obesity in Population-Based Studies in Iranian Children and Adolescents (Based on BMI Measure)

\begin{tabular}{|c|c|c|c|c|c|c|c|c|c|c|c|c|c|}
\hline Reference & Location & Urban/Rural & $\begin{array}{l}\text { Year of } \\
\text { Study }\end{array}$ & Gender & $\begin{array}{l}\text { Age- } \\
\text { group, } \\
\mathbf{y}\end{array}$ & Age $^{a}$ & $\begin{array}{c}\text { Sample } \\
\text { Size, } \mathbf{n}\end{array}$ & BMI $^{\mathbf{a}}$ & $\begin{array}{c}\text { CI 95\% } \\
\text { BMI Mean }\end{array}$ & $\begin{array}{c}\text { Obesity } \\
\text { Definition }\end{array}$ & $\begin{array}{c}\text { Prevalence } \\
\text { of } \\
\text { Obesity/CI } \\
95 \%\end{array}$ & $\begin{array}{c}\text { Over } \\
\text { Weight } \\
\text { Definition }\end{array}$ & $\begin{array}{c}\text { Prevalence } \\
\text { of Over } \\
\text { Weight/CI } \\
95 \%\end{array}$ \\
\hline \multirow{4}{*}{$\begin{array}{l}\text { Montazerifar } \\
\text { et al. } \\
(1999)(68)\end{array}$} & $\begin{array}{l}\text { Yazd, local } \\
\text { study }\end{array}$ & u & 1998 & & & & & & & CDC 2000 & & CDC 2000 & \\
\hline & & & & Both & $7-11$ & $\mathrm{NA}$ & 463 & $\mathrm{NA}$ & $\mathrm{NA}$ & & $\begin{array}{l}3.9(3.56- \\
4.24)\end{array}$ & & $\mathrm{NA}$ \\
\hline & & & & Male & & $\mathrm{NA}$ & 230 & $\mathrm{NA}$ & $\mathrm{NA}$ & & $\begin{array}{c}4.3(3.77- \\
4.83)\end{array}$ & & $\mathrm{NA}$ \\
\hline & & & & Female & & $\mathrm{NA}$ & 223 & $\mathrm{NA}$ & $\mathrm{NA}$ & & $\begin{array}{c}3.4(2.98- \\
3.82)\end{array}$ & & $\mathrm{NA}$ \\
\hline \multirow{4}{*}{$\begin{array}{l}\text { Mostafavi } \\
\text { et al. } \\
(2005) \\
(69)\end{array}$} & $\begin{array}{c}\text { Shiraz, } \\
\text { local study }\end{array}$ & $\mathrm{u}$ & 2002 & & & & & & & CDC 2000 & & CDC 2000 & \\
\hline & & & & Both & $13 \cdot 18$ & $\mathrm{NA}$ & 803 & $\mathrm{NA}$ & & & $\begin{array}{c}2.9(2.51 \text { - } \\
3.29)\end{array}$ & & $\begin{array}{c}11.3(9.91 \text { - } \\
12.69)\end{array}$ \\
\hline & & & & Male & & $\mathrm{NA}$ & $\mathrm{NA}$ & $19.4 \pm 3.4$ & $18.93-19.87$ & & $\mathrm{NA}$ & & NA \\
\hline & & & & Female & & $\mathrm{NA}$ & $\mathrm{NA}$ & $20.6 \pm 3.8$ & $20.07-21.13$ & & $\mathrm{NA}$ & & $\mathrm{NA}$ \\
\hline $\begin{array}{l}\text { Mozaffari } \\
\text { et al. } \\
(2007) \\
(70)\end{array}$ & $\begin{array}{l}\text { Tehran, } \\
\text { local study }\end{array}$ & u & 2002 & Female & $7-12$ & $9.67 \pm 3.5$ & 1800 & $\mathrm{NA}$ & $\mathrm{NA}$ & CDC 2000 & $\begin{array}{c}7.7(7.37- \\
8.03)\end{array}$ & CDC 2000 & $\begin{array}{c}13.3(12.77- \\
13.83)\end{array}$ \\
\hline \multirow{4}{*}{$\begin{array}{l}\text { Mozaffari } \\
\text { et al. } \\
(\mathbf{2 0 0 4})(71)\end{array}$} & $\begin{array}{l}\text { Yazd, local } \\
\text { study }\end{array}$ & u & 2003 & & & & & & & $\mathrm{NCHS}$ & & NCHS & \\
\hline & & & & Both & $6-12$ & $\mathrm{NA}$ & 4755 & $\mathrm{NA}$ & $\mathrm{NA}$ & & $\begin{array}{c}3.5(3.40- \\
3.60)\end{array}$ & & $\mathrm{NA}$ \\
\hline & & & & Male & & NA & 2948 & $\mathrm{NA}$ & $\mathrm{NA}$ & & $\begin{array}{c}1.0(0.96- \\
1.04)\end{array}$ & & $\mathrm{NA}$ \\
\hline & & & & Female & & $\mathrm{NA}$ & 1807 & $\mathrm{NA}$ & $\mathrm{NA}$ & & $\begin{array}{c}7.6(7.28- \\
7.92)\end{array}$ & & $\mathrm{NA}$ \\
\hline $\begin{array}{l}\text { Pourghasem } \\
\text { et al. } \\
(\mathbf{2 0 0 2})(72)\end{array}$ & $\begin{array}{c}\text { Tabriz, } \\
\text { local study }\end{array}$ & u & $\mathrm{NA}$ & Female & $14-18$ & $\mathrm{NA}$ & 1518 & $\mathrm{NA}$ & NA & CDC 2000 & $\begin{array}{c}3.6(3.43- \\
3.77)\end{array}$ & CDC 2000 & $\begin{array}{c}11.1(10.60- \\
11.59)\end{array}$ \\
\hline $\begin{array}{l}\text { Rafraf et } \\
\text { al.(2010) } \\
(73)\end{array}$ & $\begin{array}{c}\text { Tabriz, } \\
\text { local study }\end{array}$ & U & 2008 & Female & $14-17$ & $\begin{array}{c}15.67 \pm \\
1.01\end{array}$ & 985 & $21.28 \pm 3.5$ & $21.06-21.50$ & CDC 2000 & $\begin{array}{l}\text { 2. }(2.63- \\
2.97)\end{array}$ & CDC 2000 & $\begin{array}{c}16.4(15.54- \\
17.26)\end{array}$ \\
\hline \multirow{3}{*}{$\begin{array}{l}\text { Rashidi et } \\
\text { al. (2007) } \\
(74)\end{array}$} & $\begin{array}{c}\text { Tehran, } \\
\text { local study }\end{array}$ & U & 2001 & & & & & & & CDC 2000 & & CDC 2000 & \\
\hline & & & & Male & $11-16$ & $13.7 \pm 1.54$ & 1068 & $19.8 \pm 4$ & $\begin{array}{l}20.37- \\
20.83\end{array}$ & & $\begin{array}{c}7.3(6.89- \\
7.70)\end{array}$ & & $\begin{array}{c}18.8(17.88- \\
19.72)\end{array}$ \\
\hline & & & & Female & & $13.4 \pm 1.64$ & 1253 & $20.6 \pm 4.1$ & $17.75-18.05$ & & $\begin{array}{c}8.3(7.87- \\
8.72)\end{array}$ & & $\begin{array}{c}23.1(22.12- \\
24.08)\end{array}$ \\
\hline $\begin{array}{l}\text { Sadeqipoor } \\
\text { et al. } \\
(1999)(75)\end{array}$ & $\begin{array}{c}\text { Tehran, } \\
\text { local study }\end{array}$ & u & 1996 & Female & 11-14 & $\mathrm{NA}$ & 350 & $\mathrm{NA}$ & $\mathrm{NA}$ & CDC 2000 & $\begin{array}{c}13.7(12.46- \\
14.94)\end{array}$ & CDC 2000 & $\mathrm{NA}$ \\
\hline \multirow{2}{*}{$\begin{array}{l}\text { Salem et } \\
\text { al. (2009) } \\
(76)\end{array}$} & $\begin{array}{c}\text { Kerman, } \\
\text { local study }\end{array}$ & u & 2007 & & & & & & & CDC 2000 & & CDC 2000 & \\
\hline & & & & Female & $11-18$ & $14.3 \pm 1.7$ & 1221 & $\mathrm{NA}$ & $\mathrm{NA}$ & & $\begin{array}{c}\mathrm{F}: 2.4(2.27- \\
2.53)(11-14 \\
\text { yo: } 3.3)(15- \\
18 \text { yo: } 1.3)\end{array}$ & & $\begin{array}{c}\text { F: } 11.2 \\
(10.64- \\
11.76)(11-14 \\
\text { yo: } 11.9)(15- \\
18 \text { yo: } 10.8)\end{array}$ \\
\hline
\end{tabular}

Abbreviation: CI, confidence interval; F, female; $\mathrm{M}$, male; $\mathrm{NA}$, not available; $\mathrm{R}$, rural; $\mathrm{R}$, rural total; $\mathrm{T}$, total; U, urban; $\mathrm{UT}$, urban total; $\mathrm{y}$, year. ${ }^{\mathrm{a}}$ Values are expressed as mean (SD). 
Table 9. [Part 7] The Prevalence of Obesity in Population-Based Studies in Iranian Children and Adolescents (Based on BMI Measure)

\begin{tabular}{|c|c|c|c|c|c|c|c|c|c|c|c|c|c|}
\hline Reference & Location & Urban/Rural & $\begin{array}{l}\text { Year of } \\
\text { Study }\end{array}$ & Gender & $\begin{array}{l}\text { Age-group, } \\
\mathbf{y}\end{array}$ & Age ${ }^{a}$ & $\begin{array}{c}\text { Sample } \\
\text { Size, } \mathbf{n}\end{array}$ & BMI $^{\mathbf{a}}$ & $\begin{array}{c}\text { CI 95\% BMI } \\
\text { Mean }\end{array}$ & $\begin{array}{c}\text { Obesity } \\
\text { Definition }\end{array}$ & $\begin{array}{c}\text { Prevalence } \\
\text { of } \\
\text { Obesity/CI } \\
95 \%\end{array}$ & $\begin{array}{c}\text { Over } \\
\text { Weight } \\
\text { Definition }\end{array}$ & $\begin{array}{c}\text { Prevalence } \\
\text { of Over } \\
\text { Weight/CI } \\
95 \%\end{array}$ \\
\hline \multirow{3}{*}{$\begin{array}{l}\text { Salem et } \\
\text { al.(2011) } \\
\text { (77) }\end{array}$} & $\begin{array}{l}\text { Kerman, } \\
\text { local study }\end{array}$ & $\mathrm{u}$ & 2009 & & & & & & & CDC 2000 & & CDC 2000 & \\
\hline & & & & Male & $\mathrm{NA}$ & $\mathrm{NA}$ & 500 & $\begin{array}{c}16.78 \pm \\
3.76\end{array}$ & $16.45-17.11$ & & $\mathrm{NA}$ & & $\begin{array}{c}10.2(9.40- \\
11.00)\end{array}$ \\
\hline & & & & Female & $\mathrm{NA}$ & $\mathrm{NA}$ & 775 & $17.25 \pm 3.33$ & $17.02-17.48$ & & $\mathrm{NA}$ & & $\begin{array}{c}8.9(8.33- \\
9.47)\end{array}$ \\
\hline $\begin{array}{l}\text { Saffari et } \\
\text { al. (2011) } \\
\text { (78) }\end{array}$ & $\begin{array}{l}\text { Qazvin, } \\
\text { local study }\end{array}$ & $\mathrm{U}$ & 2010 & Female & $6-14$ & $\mathrm{NA}$ & 2201 & $\begin{array}{c}17.9 \pm 3.7 \\
(6-9 \text { yo: } \\
16.10)(10- \\
14 \text { yo: } 19.1(5)\end{array}$ & $\mathrm{NA}$ & CDC 2000 & $\mathrm{NA}$ & CDC 2000 & $\mathrm{NA}$ \\
\hline \multirow{4}{*}{$\begin{array}{l}\text { Sohailifar } \\
\text { et al. } \\
(\mathbf{2 0 0 0})(79)\end{array}$} & $\begin{array}{l}\text { Hamadan, } \\
\text { local study }\end{array}$ & $\mathrm{u}$ & 1998 & & & & & & & NCHS & & NCHS & \\
\hline & & & & Both & 11-16 & $\mathrm{NA}$ & 2000 & $\mathrm{NA}$ & $\mathrm{NA}$ & & $\begin{array}{c}3.5(3.35- \\
3.65)\end{array}$ & & $\mathrm{NA}$ \\
\hline & & & & Male & & $\mathrm{NA}$ & 1000 & $\mathrm{NA}$ & $\mathrm{NA}$ & & $\begin{array}{c}4(3.76- \\
4.24)\end{array}$ & & $\mathrm{NA}$ \\
\hline & & & & Female & & $\mathrm{NA}$ & 1000 & $\mathrm{NA}$ & $\mathrm{NA}$ & & $\begin{array}{c}7.2(6.79- \\
7.61)\end{array}$ & & $\mathrm{NA}$ \\
\hline $\begin{array}{l}\text { Sotoodeh } \\
\text { et al. } \\
\text { (1997) (80) }\end{array}$ & $\begin{array}{c}\text { Hassanabad } \\
\text { Khaleseh of } \\
\text { Es- } \\
\text { lamshahr, } \\
\text { local study }\end{array}$ & $\mathrm{R}$ & 1994 & Female & $15-19$ & $\mathrm{NA}$ & 35 & 22.5 & $\mathrm{NA}$ & CDC 2000 & $\mathrm{NA}$ & CDC 2000 & $\mathrm{NA}$ \\
\hline \multirow{4}{*}{$\begin{array}{l}\text { Taheri et } \\
\text { al.(2009) } \\
(81)\end{array}$} & $\begin{array}{l}\text { Birjand, } \\
\text { local study }\end{array}$ & $\mathrm{u}$ & 2005 & & & & & & & CDC 2000 & & $\mathrm{CDC} 2000$ & \\
\hline & & & & Both & $15-18$ & $\mathrm{NA}$ & 2230 & $\mathrm{NA}$ & $\mathrm{NA}$ & & $\begin{array}{l}2.2(2.11- \\
2.29)\end{array}$ & & $\begin{array}{c}6.2(5.96- \\
6.44)\end{array}$ \\
\hline & & & & Male & & $\mathrm{NA}$ & 1115 & $\mathrm{NA}$ & $\mathrm{NA}$ & & $\begin{array}{l}2.8(2.64 \text { - } \\
2.96)\end{array}$ & & $\begin{array}{l}5(4.72- \\
5.28)\end{array}$ \\
\hline & & & & Female & & $\mathrm{NA}$ & 1115 & $\mathrm{NA}$ & $\mathrm{NA}$ & & $\begin{array}{c}1.8(1.70- \\
1.90)\end{array}$ & & $\begin{array}{c}7.1(6.71- \\
7.49)\end{array}$ \\
\hline \multirow{4}{*}{$\begin{array}{l}\text { Taheri et } \\
\text { al. (2013) } \\
\text { (82) }\end{array}$} & $\begin{array}{l}\text { Birjand, } \\
\text { local study }\end{array}$ & u & 2012 & & & & & & & CDC 2000 & & CDC 2000 & \\
\hline & & & & Both & $6 \cdot 11$ & $\mathrm{NA}$ & 1541 & $\mathrm{NA}$ & $\mathrm{NA}$ & & $\begin{array}{l}9.2(8.78- \\
9.62)\end{array}$ & & $\begin{array}{c}9.6(9.17- \\
10.03)\end{array}$ \\
\hline & & & & & & $\mathrm{NA}$ & 690 & 16.9 & $\mathrm{NA}$ & & $\begin{array}{c}10.9(10.18- \\
11.62)\end{array}$ & & $\begin{array}{c}11(10.27- \\
11.73)\end{array}$ \\
\hline & & & & & & $\mathrm{NA}$ & 851 & 16.3 & $\mathrm{NA}$ & & $\begin{array}{c}7.9(7.41- \\
8.39)\end{array}$ & & $\begin{array}{c}8.3(7.79- \\
8.81)\end{array}$ \\
\hline $\begin{array}{l}\text { Veghari et } \\
\text { al. (2010) } \\
(83)\end{array}$ & $\begin{array}{l}\text { Golestan, } \\
\text { local study }\end{array}$ & Both & 2006 & Both & $15-24$ & $\mathrm{NA}$ & 499 & 22.8 & $22.3-23.4$ & CDC 2000 & $\begin{array}{l}5.1(4.67- \\
5.52)\end{array}$ & CDC 2000 & $\begin{array}{c}24.2(22.59- \\
25.81)\end{array}$ \\
\hline
\end{tabular}

Abbreviation: $\mathrm{CI}$, confidence interval; $\mathrm{F}$, female; $\mathrm{M}$, male; $\mathrm{NA}$, not available; $\mathrm{R}$, rural; $\mathrm{R}$, rural total; $\mathrm{T}$, total; $\mathrm{U}$, urban; $\mathrm{UT}$, urban total; $\mathrm{y}$, year. ${ }^{\mathrm{a}}$ Values are expressed as mean (SD). 
Table 10. [Part 8] The Prevalence of Obesity in Population-Based Studies in Iranian Children and Adolescents (Based on BMI Measure)

\begin{tabular}{|c|c|c|c|c|c|c|c|c|c|c|c|c|c|}
\hline Reference & Location & Urban/Rural & $\begin{array}{l}\text { Year of } \\
\text { Study }\end{array}$ & Gender & $\begin{array}{l}\text { Age- } \\
\text { group, } \\
\mathbf{y}\end{array}$ & Age & $\begin{array}{c}\text { Sample } \\
\text { Size, } \mathbf{n}\end{array}$ & BMI & $\begin{array}{c}\text { CI 95\% } \\
\text { BMI Mean }\end{array}$ & $\begin{array}{c}\text { Obesity } \\
\text { Definition }\end{array}$ & $\begin{array}{c}\text { Prevalence } \\
\text { of } \\
\text { Obesity/CI } \\
95 \%\end{array}$ & $\begin{array}{c}\text { Over } \\
\text { Weight } \\
\text { Definition }\end{array}$ & $\begin{array}{c}\text { Prevalence } \\
\text { of Over } \\
\text { Weight/CI } \\
95 \%\end{array}$ \\
\hline \multirow{4}{*}{$\begin{array}{l}\text { Veghari et } \\
\text { al.(2012) } \\
(84)\end{array}$} & $\begin{array}{l}\text { Golestan, } \\
\text { local study }\end{array}$ & Both & 2008 & & & & & & & CDC 2000 & & CDC 2000 & \\
\hline & & & & Both & $15-65$ & $39.6 \pm 14.3$ & 6489: & $\mathrm{NA}$ & $\mathrm{NA}$ & & $\begin{array}{c}23(22.39 \text { - } \\
23.61)\end{array}$ & & $\begin{array}{c}31.5(30.76- \\
32.2)\end{array}$ \\
\hline & & & & Male & & $\mathrm{NA}$ & 3245 & $25 \pm 4.8$ & $24.88-25.12$ & & $\begin{array}{c}14.1(13.68- \\
14.52)\end{array}$ & & $\begin{array}{c}32.4(31.65- \\
33.15)\end{array}$ \\
\hline & & & & Female & & $\mathrm{NA}$ & 3244 & $27.5 \pm 6.1$ & $27.29-27.71$ & & $\begin{array}{l}31.8(31.27- \\
32.33)\end{array}$ & & $\begin{array}{c}30.5(29.98 \\
-31.02)\end{array}$ \\
\hline \multirow[t]{3}{*}{$\begin{array}{l}\text { Kelishadi } \\
\text { et al. }(85)\end{array}$} & $\begin{array}{l}\text { CASPIAN I, } \\
\text { national } \\
\text { study }\end{array}$ & Both & 2004 & & & & & & & CDC 2000 & & CDC 2000 & \\
\hline & & & & Both & $6-18$ & $\begin{array}{c}12.27 \pm \\
3.32\end{array}$ & 20,966 & $\begin{array}{c}18.42 \pm \\
3.87\end{array}$ & $18.42-3.8$ & & $\begin{array}{c}3.42(3.17- \\
3.67)\end{array}$ & & $\begin{array}{c}10.96 \\
(10.54- \\
11.39)\end{array}$ \\
\hline & & & & Female & & $\begin{array}{c}12.27 \pm \\
3.34\end{array}$ & 10,173 & $\begin{array}{c}18.60 \pm \\
3.91\end{array}$ & $\begin{array}{c}18.60 \pm \\
3.91\end{array}$ & & $\begin{array}{c}3.50(3.15- \\
3.87)\end{array}$ & & $\begin{array}{c}12.30(11.66 \\
-12.95)\end{array}$ \\
\hline \multirow[t]{4}{*}{$\begin{array}{l}\text { Kelishadi } \\
\text { et al. (30) }\end{array}$} & $\begin{array}{l}\text { CASPIAN } \\
\text { III, } \\
\text { national } \\
\text { study }\end{array}$ & Both & 2010 & & & & & & & CDC 2000 & & CDC 2000 & \\
\hline & & & & Both & $10-18$ & $14.27 \pm 24$ & 5625 & $\begin{array}{c}19.42 \pm \\
4.09\end{array}$ & $19.31-19.53$ & & $\begin{array}{c}8.91(8.17- \\
9.68)\end{array}$ & & $\begin{array}{c}8.02(7.32- \\
8.75)\end{array}$ \\
\hline & & & & Male & & $14.68 \pm 24$ & 2824 & $\begin{array}{c}19.61 \pm \\
4.12\end{array}$ & $19.45-19.76$ & & $\begin{array}{c}10.13(9.03- \\
11.29)\end{array}$ & & $\begin{array}{c}9.38(8.33- \\
10.51)\end{array}$ \\
\hline & & & & Female & & $14.76 \pm 23$ & 2801 & $\begin{array}{c}19.23 \pm \\
4.06\end{array}$ & $19.08-19.38$ & & $\begin{array}{c}7.68(6.71- \\
8.72)\end{array}$ & & $\begin{array}{c}6.64(5.74- \\
7.62)\end{array}$ \\
\hline \multirow[t]{3}{*}{$\begin{array}{l}\text { Khashayar } \\
\text { et al. } \\
\text { (2013) (86) }\end{array}$} & $\begin{array}{c}\text { CASPIAN IV, } \\
\text { national } \\
\text { study }\end{array}$ & Both & 2012 & & & & & & & $\mathrm{CDC} 2000$ & & CDC 2000 & \\
\hline & & & & Both & $6-18$ & $\begin{array}{c}12.47 \pm \\
3.36\end{array}$ & 13,350 & $\begin{array}{c}18.85 \pm \\
4.42\end{array}$ & $18.70-18.99$ & & $\begin{array}{c}11.89(11.22- \\
12.59)\end{array}$ & & $\begin{array}{c}9.66(9.13- \\
10.21)\end{array}$ \\
\hline & & & & Female & & $\begin{array}{c}12.58 \pm \\
3.32\end{array}$ & 6573 & $\begin{array}{c}18.97 \pm \\
4.39\end{array}$ & $18.76-19.17$ & & $\begin{array}{c}10.15(9.27 . \\
11.09)\end{array}$ & & $\begin{array}{c}10.06(9.30 \\
-10.86)\end{array}$ \\
\hline
\end{tabular}

Abbreviation: CI, confidence interval; F, female; $\mathrm{M}$, male; $\mathrm{NA}$, not available; $\mathrm{R}$, rural; $\mathrm{R}$, rural total; $\mathrm{T}$, total; $\mathrm{U}$, urban; $\mathrm{UT}$, urban total; $\mathrm{y}$, year-

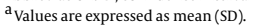


Table 11. [Part 1] The WHR, WC, and WHtR Mean and Abdominal Obesity in Population-Based Studies in Iranian Children and Adolescents

\begin{tabular}{|c|c|c|c|c|c|c|c|c|c|c|c|c|c|c|}
\hline Reference & Location & Urban/Rural & $\begin{array}{l}\text { Year of } \\
\text { Study }\end{array}$ & Gender & $\begin{array}{c}\text { Age- } \\
\text { group,y }\end{array}$ & $\mathrm{Age}^{\mathrm{a}}$ & $\begin{array}{l}\text { Sample } \\
\text { Size, n }\end{array}$ & $\mathrm{WHR}^{\mathrm{a}}$ & $\begin{array}{l}\text { CI 95\% } \\
\text { WHR } \\
\text { Mean }\end{array}$ & $\begin{array}{c}\text { Prevalence } \\
\text { of Ab- } \\
\text { dominal } \\
\text { Obesity, } \\
\%\end{array}$ & $w^{a}$ & $\begin{array}{l}\text { Cl 95\% } \\
\text { WC } \\
\text { mean }\end{array}$ & $\mathrm{WH}^{\mathrm{a}} \mathrm{R}^{\mathrm{a}}$ & $\begin{array}{l}\text { CI 95\% } \\
\text { WHtR } \\
\text { Mean }\end{array}$ \\
\hline \multirow{4}{*}{$\begin{array}{l}\text { Amini et } \\
\text { al.(2007) } \\
\text { (41) }\end{array}$} & Tehran & $\mathrm{u}$ & & & & & & & & & & & & \\
\hline & & & 2001 & Both & $10-15$ & $\begin{array}{c}12.6 \pm \\
0.9\end{array}$ & 395 & $\begin{array}{c}0.8 \pm \\
0.07\end{array}$ & $0.79-0.81$ & $\mathrm{NA}$ & $\mathrm{NA}$ & $\mathrm{NA}$ & $\mathrm{NA}$ & $\mathrm{NA}$ \\
\hline & & & 2001 & Male & & $\begin{array}{c}12.7 \pm \\
0.9\end{array}$ & 197 & $\begin{array}{c}0.83 \pm \\
0.06\end{array}$ & $0.82-0.84$ & $\mathrm{NA}$ & $\mathrm{NA}$ & $\mathrm{NA}$ & $\mathrm{NA}$ & $\mathrm{NA}$ \\
\hline & & & 2001 & Female & & $\begin{array}{c}12.5 \pm \\
0.9\end{array}$ & 198 & $\begin{array}{c}0.77 \pm \\
0.06\end{array}$ & $0.76-0.78$ & $\mathrm{NA}$ & $\mathrm{NA}$ & $\mathrm{NA}$ & $\mathrm{NA}$ & $\mathrm{NA}$ \\
\hline \multirow{4}{*}{$\begin{array}{l}\text { Azimi- } \\
\text { Nezhad } \\
\text { et al. } \\
(2009) \\
(44)\end{array}$} & $\begin{array}{c}\text { Khorasan- } \\
\text { Razavi }\end{array}$ & Both & & & & & & & & & & & & \\
\hline & & & 2004 & Both & $15-20$ & $\mathrm{NA}$ & 540 & $\mathrm{NA}$ & $\mathrm{NA}$ & $\mathrm{NA}$ & $\mathrm{NA}$ & $\mathrm{NA}$ & $\mathrm{NA}$ & $\mathrm{NA}$ \\
\hline & & & 2004 & Male & $\mathrm{NA}$ & $\mathrm{NA}$ & 280 & $\mathrm{NA}$ & $\mathrm{NA}$ & $\mathrm{NA}$ & $\begin{array}{c}74.63 \pm \\
12.54\end{array}$ & $73.16-76.1$ & $\begin{array}{c}0.43 \pm \\
0.07\end{array}$ & $\begin{array}{l}0.42- \\
0.44\end{array}$ \\
\hline & & & 2004 & Female & $\mathrm{NA}$ & $\mathrm{NA}$ & 260 & $\mathrm{NA}$ & $\mathrm{NA}$ & $\mathrm{NA}$ & $\begin{array}{c}70.09 \pm \\
10.6\end{array}$ & $\begin{array}{c}68.80- \\
71.38\end{array}$ & $\begin{array}{c}0.47 \pm \\
0.06\end{array}$ & $\begin{array}{c}0.46- \\
0.48\end{array}$ \\
\hline \multirow{3}{*}{$\begin{array}{l}\text { Gharakhanlou } \\
\text { et al. } \\
\text { (2012) } \\
(49)\end{array}$} & $\begin{array}{l}\text { National } \\
\text { study }\end{array}$ & $\mathrm{U}$ & & & & & & & & & & & & \\
\hline & & & 2011 & Male & $15-19$ & $\mathrm{NA}$ & 139 & $\begin{array}{c}0.82 \pm \\
0.05\end{array}$ & $0.81-0.83$ & $\mathrm{NA}$ & $\begin{array}{c}-74.5 \pm \\
8.9\end{array}$ & $\begin{array}{c}73.02- \\
75.98\end{array}$ & $\begin{array}{c}0.43 \pm \\
0.06\end{array}$ & $0.42-0.43$ \\
\hline & & & 2011 & Female & $\mathrm{NA}$ & $\mathrm{NA}$ & 145 & $\begin{array}{c}0.78 \pm \\
0.06\end{array}$ & $0.77-0.79$ & $\mathrm{NA}$ & $\begin{array}{c}7.2 .7 \pm \\
9.0\end{array}$ & $\begin{array}{c}71.24 \text { - } \\
74.16\end{array}$ & $\begin{array}{c}0.45 \pm \\
0.07\end{array}$ & $\begin{array}{l}0.44- \\
0.46\end{array}$ \\
\hline \multirow{13}{*}{$\begin{array}{l}\text { Hosseini- } \\
\text { Esfahani } \\
\text { et al. } \\
\text { (2011) } \\
(87)\end{array}$} & Tehran & $\mathrm{u}$ & & & & & & & & & & & & \\
\hline & & & 2001 & Male & $10-14$ & $12.4 \pm 1$ & 688 & $\mathrm{NA}$ & $\mathrm{NA}$ & $\mathrm{NA}$ & $\mathrm{NA}$ & $\begin{array}{c}64.9 \pm \\
117\end{array}$ & $\begin{array}{c}64.08- \\
65.72\end{array}$ & $\mathrm{NA}$ \\
\hline & & & 2001 & Male & $15-19$ & $16.8 \pm 1$ & 734 & $\mathrm{NA}$ & $\mathrm{NA}$ & $\mathrm{NA}$ & $\mathrm{NA}$ & $3.9 \pm 11$ & $\begin{array}{l}73.10- \\
74.70\end{array}$ & $\mathrm{NA}$ \\
\hline & & & 2005 & Male & $10-14$ & $12.2 \pm 1$ & 190 & $\mathrm{NA}$ & $\mathrm{NA}$ & $\mathrm{NA}$ & $\mathrm{NA}$ & $72.6 \pm 13$ & $\begin{array}{l}70.75- \\
74.45\end{array}$ & $\mathrm{NA}$ \\
\hline & & & 2005 & Male & $15-19$ & $17.1 \pm 1$ & 346 & $\mathrm{NA}$ & $\mathrm{NA}$ & $\mathrm{NA}$ & $\mathrm{NA}$ & $80.4 \pm 12$ & $\begin{array}{c}79.14- \\
81.66\end{array}$ & $\mathrm{NA}$ \\
\hline & & & 2008 & Male & $10-14$ & $12.2 \pm 1$ & 230 & $\mathrm{NA}$ & $\mathrm{NA}$ & $\mathrm{NA}$ & $\mathrm{NA}$ & $71.3 \pm 13$ & $\begin{array}{l}69.62- \\
72.98\end{array}$ & $\mathrm{NA}$ \\
\hline & & & 2008 & Male & $15-19$ & $17.2 \pm 1$ & 287 & $\mathrm{NA}$ & $\mathrm{NA}$ & $\mathrm{NA}$ & $\mathrm{NA}$ & $81.5 \pm 13$ & $\begin{array}{c}79.97 \\
83.03\end{array}$ & $\mathrm{NA}$ \\
\hline & & & 2001 & Female & $10-14$ & $12.3 \pm 1$ & 675 & $\mathrm{NA}$ & $\mathrm{NA}$ & $\mathrm{NA}$ & $\mathrm{NA}$ & $66.9 \pm 9$ & $\begin{array}{l}66.22- \\
67.58\end{array}$ & $\mathrm{NA}$ \\
\hline & & & 2001 & Female & $15-19$ & $17.0 \pm 1$ & 913 & $\mathrm{NA}$ & $\mathrm{NA}$ & $\mathrm{NA}$ & $\mathrm{NA}$ & $72.5 \pm 9$ & $\begin{array}{l}71.92- \\
73.08\end{array}$ & $\mathrm{NA}$ \\
\hline & & & 2005 & Female & $10-14$ & $12.3 \pm 1$ & 214 & $\mathrm{NA}$ & $\mathrm{NA}$ & $\mathrm{NA}$ & $\mathrm{NA}$ & $68.5 \pm 10$ & $\begin{array}{l}67.16- \\
69.84\end{array}$ & $\mathrm{NA}$ \\
\hline & & & 2005 & Female & $15-19$ & $17.3 \pm 1$ & 357 & $\mathrm{NA}$ & $\mathrm{NA}$ & $\mathrm{NA}$ & $\mathrm{NA}$ & $73.3 \pm 9$ & $\begin{array}{l}72.37- \\
74.23\end{array}$ & $\mathrm{NA}$ \\
\hline & & & 2008 & Female & $10-14$ & $12.3 \pm 1$ & 231 & $\mathrm{NA}$ & $\mathrm{NA}$ & $\mathrm{NA}$ & $\mathrm{NA}$ & $66.9 \pm 10$ & $\begin{array}{l}65.61- \\
68.19\end{array}$ & $\mathrm{NA}$ \\
\hline & & & 2008 & Female & $15-19$ & $17.2 \pm 1$ & 351 & $\mathrm{NA}$ & $\mathrm{NA}$ & $\mathrm{NA}$ & $\mathrm{NA}$ & $71.3 \pm 9$ & $\begin{array}{c}70.36- \\
72.24\end{array}$ & $\mathrm{NA}$ \\
\hline
\end{tabular}

Abbreviation: $\mathrm{Cl}$, confidence interval; $\mathrm{F}$, female; $\mathrm{M}$, male; $\mathrm{NA}$, not available; $\mathrm{R}$, rural; $\mathrm{R}$, rural total; $\mathrm{T}$, total; $\mathrm{U}$, urban; UT, urban total; $\mathrm{y}$, year. ${ }^{\mathrm{a}}$ Values are expressed as mean (SD). 
Table 12. [Part 2] The WHR, WC, and WHtR Mean and Abdominal Obesity in Population-Based Studies in Iranian Children and Adolescents

\begin{tabular}{|c|c|c|c|c|c|c|c|c|c|c|c|c|c|c|}
\hline Reference & Location & Urban/Rural & $\begin{array}{l}\text { Year of } \\
\text { Study }\end{array}$ & Gender & $\begin{array}{c}\text { Age- } \\
\text { group, } y\end{array}$ & Age & $\begin{array}{c}\text { Sample } \\
\text { Size, } \mathbf{n}\end{array}$ & WHR & $\begin{array}{l}\text { CI 95\% } \\
\text { WHR } \\
\text { Mean }\end{array}$ & $\begin{array}{c}\text { Prevalence } \\
\text { of Ab- } \\
\text { dominal } \\
\text { Obesity, } \%\end{array}$ & wc & $\begin{array}{c}\text { CI 95\% } \\
\text { WC mean }\end{array}$ & WHtR & $\begin{array}{l}\text { CI 95\% } \\
\text { WHtR } \\
\text { Mean }\end{array}$ \\
\hline $\begin{array}{l}\text { Janghorbani } \\
\text { et al. } \\
\text { (1998) } \\
(54)\end{array}$ & Kerman & U & 1995 & Female & $14-18$ & $16.2 \pm 1.3$ & 1000 & $\begin{array}{c}0.8 \pm \\
0.06\end{array}$ & $0.8-0.81$ & $\mathrm{NA}$ & $\mathrm{NA}$ & $\begin{array}{c}70.8 \pm \\
8.0\end{array}$ & $70-71.0$ & $\mathrm{NA}$ \\
\hline \multirow{3}{*}{$\begin{array}{l}\text { Janghorbani } \\
\text { et al. } \\
(2007) \\
(88)\end{array}$} & National & Both & & & & & & & & & & & & \\
\hline & & & 2005 & Male & $15-24$ & $\begin{array}{c}23.9 \pm \\
0.15\end{array}$ & v & $\mathrm{NA}$ & $\mathrm{NA}$ & 3.2 & $\mathrm{NA}$ & $\mathrm{NA}$ & NA & $\mathrm{NA}$ \\
\hline & & & 2005 & Female & $\mathrm{NA}$ & $\begin{array}{c}24.9 \pm \\
0.18\end{array}$ & $\mathrm{NA}$ & $\mathrm{NA}$ & $\mathrm{NA}$ & 18.1 & - & . & - & - \\
\hline \multirow{3}{*}{$\begin{array}{l}\text { Mehrkash } \\
\text { et al. } \\
(2010) \\
(61)\end{array}$} & $\begin{array}{c}\text { National } \\
\text { study }\end{array}$ & $\mathrm{u}$ & & & & & & & & & & & & \\
\hline & & & $\mathrm{NA}$ & Both & $15-18$ & $\begin{array}{c}15.66 \pm \\
0.68\end{array}$ & 225 & $\mathrm{NA}$ & $\mathrm{NA}$ & $\mathrm{NA}$ & NA & $\begin{array}{c}75.24 \pm \\
8.76\end{array}$ & $\begin{array}{l}74.43- \\
76.05\end{array}$ & $\mathrm{NA}$ \\
\hline & & & $\mathrm{NA}$ & Both & NA & $\begin{array}{c}16.34 \pm \\
0.77\end{array}$ & 225 & $\mathrm{NA}$ & $\mathrm{NA}$ & $\mathrm{NA}$ & $\mathrm{NA}$ & $\begin{array}{c}79.85 \pm \\
10.35\end{array}$ & $\begin{array}{c}78.50- \\
81.20\end{array}$ & $\mathrm{NA}$ \\
\hline $\begin{array}{l}\text { Mirhosseini } \\
\text { et al. } \\
(2009) \\
(62)\end{array}$ & $\begin{array}{l}\text { Mashhad, } \\
\text { Local } \\
\text { study }\end{array}$ & $\mathrm{U}$ & $\mathrm{NA}$ & Female & $15-17$ & $\begin{array}{c}16.4 \pm \\
0.09\end{array}$ & 622 & $\begin{array}{c}0.7 \pm \\
0.04\end{array}$ & $0.69-0.70$ & 9.5 & $\mathrm{NA}$ & $69 \pm 7.8$ & $\begin{array}{l}68.39- \\
69.61\end{array}$ & $\mathrm{NA}$ \\
\hline \multirow[t]{2}{*}{$\begin{array}{l}\text { Sotoodeh } \\
\text { et al. }(80)\end{array}$} & $\begin{array}{l}\text { Hassanabad } \\
\text { of Es- } \\
\text { lamshahr, } \\
\text { Local } \\
\text { study }\end{array}$ & alesehR & & & & & & & & & & & & \\
\hline & & & 1994 & Female & $15-19$ & $\mathrm{NA}$ & 205 & 0.78 & NA & $\mathrm{NA}$ & $\mathrm{NA}$ & $\mathrm{NA}$ & $\mathrm{NA}$ & $\mathrm{NA}$ \\
\hline
\end{tabular}

Abbreviation: $\mathrm{Cl}$, confidence interval; $\mathrm{F}$, female; $\mathrm{M}$, male; $\mathrm{NA}$, not available; $\mathrm{R}$, rural; $\mathrm{R}$, rural total; $\mathrm{T}$, total; $\mathrm{U}$, urban; $\mathrm{UT}$, urban total; $\mathrm{y}$, year.
$\mathrm{a}_{\text {Values are expressed as mean }(\mathrm{SD}) \text {. }}$ 
Table 13. [Part 3] The WHR, WC, and WHtR Mean and Abdominal Obesity in Population-Based Studies in Iranian Children and Adolescents

\begin{tabular}{|c|c|c|c|c|c|c|c|c|c|c|c|c|c|c|}
\hline Reference & Location & Urban/Rural & $\begin{array}{l}\text { Year of } \\
\text { Study }\end{array}$ & Gender & $\begin{array}{l}\text { Age- } \\
\text { group, y }\end{array}$ & Age & $\begin{array}{c}\text { Sample } \\
\text { Size, } \mathbf{n}\end{array}$ & WHR & $\begin{array}{l}\text { CI 95\% } \\
\text { WHR } \\
\text { Mean }\end{array}$ & $\begin{array}{c}\text { Prevalence } \\
\text { of Ab- } \\
\text { dominal } \\
\text { Obesity, } \\
\%\end{array}$ & wc & $\begin{array}{c}\text { CI 95\% } \\
\text { WC } \\
\text { mean }\end{array}$ & WHtR & $\begin{array}{l}\text { Cl 95\% } \\
\text { WHtR } \\
\text { Mean }\end{array}$ \\
\hline \multirow{3}{*}{$\begin{array}{l}\text { Kelishadi } \\
\text { et al. } \\
(1997) \\
(85)\end{array}$} & & & 2004 & Both & 6-18 & $\begin{array}{c}12.27 \pm \\
3.32\end{array}$ & 20,966 & $\begin{array}{c}0.43 \pm \\
0.06\end{array}$ & $\begin{array}{c}0.429- \\
0.431\end{array}$ & $\begin{array}{l}9.27(8.87- \\
9.67)\end{array}$ & $\begin{array}{c}64.61 \pm \\
10.91\end{array}$ & $\begin{array}{l}64.46- \\
64.76\end{array}$ & $\begin{array}{c}0.80 \pm \\
0.08\end{array}$ & $\begin{array}{l}0.805- \\
0.807\end{array}$ \\
\hline & & & 2004 & Male & $\mathrm{NA}$ & $\begin{array}{c}12.27 \pm \\
3.3\end{array}$ & 10,793 & $\begin{array}{c}0.43 \pm \\
0.06\end{array}$ & $\begin{array}{l}0.427 . \\
0.429\end{array}$ & $\begin{array}{c}9.14(8.60- \\
9.69)\end{array}$ & $\begin{array}{c}64.99 \pm \\
11.41\end{array}$ & $\begin{array}{r}64.78- \\
65.21\end{array}$ & $\begin{array}{c}0.82 \pm \\
0.09\end{array}$ & $\begin{array}{l}0.817- \\
0.821\end{array}$ \\
\hline & & & 2004 & Female & $\mathrm{NA}$ & $\begin{array}{c}12.27 \pm \\
3.34\end{array}$ & 10,173 & $\begin{array}{c}0.44 \pm \\
0.06\end{array}$ & $\begin{array}{l}0.431- \\
0.433\end{array}$ & $\begin{array}{l}9.41(8.84- \\
9.99)\end{array}$ & $\begin{array}{c}64.21 \pm \\
10.34\end{array}$ & $\begin{array}{l}64.01- \\
64.41\end{array}$ & $\begin{array}{c}0.79 \pm \\
0.08\end{array}$ & $\begin{array}{l}0.791- \\
0.794\end{array}$ \\
\hline \multirow[t]{4}{*}{$\begin{array}{l}\text { Kelishadi } \\
\text { (2012)et } \\
\text { al. (30) }\end{array}$} & $\begin{array}{l}\text { CASPIAN } \\
\text { III, } \\
\text { National } \\
\text { study }\end{array}$ & Both & & & & & & & & & & & & \\
\hline & & & 2010 & Both & $10-18$ & $\begin{array}{c}14.27 \pm \\
24\end{array}$ & 5625 & $\begin{array}{c}0.44 \pm \\
0.13\end{array}$ & $\begin{array}{c}0.442- \\
0.448\end{array}$ & $\mathrm{NA}$ & $\begin{array}{c}68.72 \pm \\
20.67\end{array}$ & $68.1-69.2$ & $\mathrm{NA}$ & $\mathrm{NA}$ \\
\hline & & & 2010 & Male & $\mathrm{NA}$ & $\begin{array}{c}14.68 \pm \\
24\end{array}$ & 2824 & $\begin{array}{c}0.44 \pm \\
0.14\end{array}$ & $\begin{array}{l}0.439- \\
0.449\end{array}$ & $\mathrm{NA}$ & $\begin{array}{c}67.59 \pm \\
22.16\end{array}$ & $66.7-68.4$ & $\mathrm{NA}$ & $\mathrm{NA}$ \\
\hline & & & 2010 & Female & $\mathrm{NA}$ & $\begin{array}{c}14.76 \pm \\
23\end{array}$ & 2801 & $\begin{array}{c}0.44 \pm \\
0.12\end{array}$ & $\begin{array}{l}0.441 \\
0.450\end{array}$ & $\mathrm{NA}$ & $\begin{array}{c}69.86 \pm \\
18.99\end{array}$ & $69.1-70.5$ & $\mathrm{NA}$ & $\mathrm{NA}$ \\
\hline \multirow[t]{4}{*}{$\begin{array}{l}\text { Khashayar } \\
\text { (2013) et } \\
\text { al. (86) }\end{array}$} & $\begin{array}{l}\text { CASPIAN } \\
\text { IV, } \\
\text { National } \\
\text { study }\end{array}$ & Both & & & & & & & & & & & & \\
\hline & & & 2012 & Both & $6-18$ & $\begin{array}{c}12.47 \pm \\
3.36\end{array}$ & 13,350 & $\begin{array}{c}0.45 \pm \\
0.06\end{array}$ & $\begin{array}{c}0.454- \\
0.458\end{array}$ & $\begin{array}{l}\text { 19.12(18.22- } \\
20.06)\end{array}$ & $\begin{array}{c}67.02 \pm \\
11.96\end{array}$ & $\begin{array}{l}66.57- \\
67.48\end{array}$ & $\begin{array}{c}0.18 \pm \\
0.03\end{array}$ & $\begin{array}{c}0.184 \text { - } \\
0.187\end{array}$ \\
\hline & & & 2012 & Male & & $\begin{array}{c}12.36 \pm \\
3.39\end{array}$ & 6777 & $\begin{array}{c}0.46 \pm \\
0.06\end{array}$ & $\begin{array}{c}0.456- \\
0.461\end{array}$ & $\begin{array}{c}20.41(19.09- \\
21.79)\end{array}$ & $\begin{array}{c}67.83 \pm \\
12.84\end{array}$ & $\begin{array}{l}67.15- \\
68.51\end{array}$ & $\begin{array}{c}0.19 \pm \\
0.03\end{array}$ & $\begin{array}{r}0.189 \text { - } \\
0.192\end{array}$ \\
\hline & & & 2012 & Female & & $\begin{array}{c}12.58 \pm \\
3.32\end{array}$ & 6573 & $\begin{array}{c}0.45 \pm \\
0.06\end{array}$ & $\begin{array}{c}0.452 \text { - } \\
0.457\end{array}$ & $\begin{array}{c}\text { 17.79(16.56- } \\
19.09)\end{array}$ & $\begin{array}{c}66.19 \pm \\
10.92\end{array}$ & $\begin{array}{l}65.62- \\
66.76\end{array}$ & $\begin{array}{c}0.18 \pm \\
0.03\end{array}$ & $\begin{array}{c}0.179 \text { - } \\
0.183\end{array}$ \\
\hline
\end{tabular}

Abbreviation: CI, confidence interval; F, female; M, male; NA, not available; $\mathrm{R}$, rural; $\mathrm{R}$, rural total; $\mathrm{T}$, total; $\mathrm{U}$, urban; UT, urban total; $\mathrm{y}$, year.

a Values are expressed as mean (SD). 


\section{Acknowledgments}

The study is granted by Setad-e-Ejraie Farmane Imam and ministry of health and medical education of Islamic Republic of Iran.

\section{Footnote}

Authors' Contribution: Shirin Djalalinia and Mostafa Qorbani had equal contribution in designing the paper, acquisition of data and data analysis, and interpretation. Niloofar Peykari, Ensieh Nasli-Esfahani, and Shohreh Naderimagham had cooperation in drafting of the manuscript and Roya Kelishadi, Bagher Larijani, and Farshad Farzadfar supervised the project and had critical revision of the manuscript and approval of the article. All authors have given approval to the final version of manuscript.

\section{References}

1. Lim SS, Vos T, Flaxman AD, Danaei G, Shibuya K, Adair-Rohani H, et al. A comparative risk assessment of burden of disease and injury attributable to 67 risk factors and risk factor clusters in 21 regions, 19902010: a systematic analysis for the Global Burden of Disease Study 2010. Lancet. 2012;380(9859):2224-60. [PubMed: 23245609].

2. Murray CJ, Ezzati M, Flaxman AD, Lim S, Lozano R, Michaud C, et al. GBD 2010: a multi-investigator collaboration for global comparative descriptive epidemiology. Lancet. 2012;380(9859):2055-8. [PubMed: 23245598].

3. Murray CJ, Lopez AD. Alternative projections of mortality and disability by cause 1990-2020: Global Burden of Disease Study. Lancet. 1997;349(9064):1498-504. [PubMed: 9167458].

4. Murray CJ, Lopez AD. Global mortality, disability, and the contribution of risk factors: Global Burden of Disease Study. Lancet. 1997;349(9063):1436-42. [PubMed: 9164317].

5. Lopez AD. The evolution of the Global Burden of Disease framework for disease, injury and risk factor quantification: developing the evidence base for national, regional and global public health action. Global Health. 2005;1(5):1-8.

6. Ezzati M, Lopez AD, Rodgers A, Vander Hoorn S, Murray CJ, Comparative Risk Assessment Collaborating G. Selected major risk factors and global and regional burden of disease. Lancet. 2002;360(9343):134760. [PubMed: 12423980].

7. Finucane MM, Stevens GA, Cowan MJ, Danaei G, Lin JK, Paciorek CJ, et al. National, regional, and global trends in body-mass index since 1980: systematic analysis of health examination surveys and epidemiological studies with 960 country-years and 9.1 million participants. Lancet. 2011;377(9765):557-67. [PubMed: 21295846].

8. Stevens GA, Singh GM, Lu Y, Danaei G, Lin JK, Finucane MM, et al. National, regional, and global trends in adult overweight and obesity prevalences. Popul Health Metr. 2012;10(1):22. [PubMed: 23167948].

9. Mirzazadeh A, Sadeghirad B, Haghdoost AA, Bahreini F, Rezazadeh Kermani M. The prevalence of obesity in Iran in recent decade; a systematic review and meta-analysis study. Iran J Public Health. 2009;38(3):1-11.

10. Dastgiri S, Mahdavi R, TuTunchi H, Faramarzi E. Prevalence of obesity, food choices and socio-economic status: a cross-sectional study in the north-west of Iran. Public Health Nutr. 2006;9(8):996-1000. [PubMed: $17125562]$
11. Musaiger AO. Overweight and obesity in eastern mediterranean region: prevalence and possible causes. J Obes. 2011;2011:407237. [PubMed: 21941635].

12. Djalalinia S, Moghaddam SS, Peykari N, Kasaeian A, Sheidaei A, Mansouri A, et al. Mortality Attributable to Excess Body Mass Index in Iran: Implementation of the Comparative Risk Assessment Methodology. Int J Prev Med. 2015;6:107. [PubMed: 26644906].

13. Bann D, Wills A, Cooper R, Hardy R, Aihie Sayer A, Adams J, et al. Birth weight and growth from infancy to late adolescence in relation to fat and lean mass in early old age: findings from the MRC National Survey of Health and Development. Int J Obes (Lond). 2014;38(1):69-75. [PubMed: 23779050].

14. Peykari N, Tehrani FR, Eftekhari MB, Malekafzali H, Dejman M, Neot $\mathrm{R}$, et al. A peer-based study on adolescence nutritional health: a lesson learned from Iran. J Pak Med Assoc. 2011;61(6):549-54. [PubMed: 22204208]

15. Cali AM, Caprio S. Obesity in children and adolescents. JClin Endocrinol Metab. 2008;93(11 Suppl 1):S31-6. [PubMed: 18987268]

16. Djalalinia S, Qorbani M, Peykari N, Kelishadi R. Health impacts of Obesity. PakJ Med Sci. 2015;31(1):239-42. [PubMed: 25878654].

17. Reilly JJ, Kelly J. Long-term impact of overweight and obesity in childhood and adolescence on morbidity and premature mortality in adulthood: systematic review. Int J Obes (Lond). 2011;35(7):891-8. [PubMed: 20975725].

18. Mier N, Smith ML, Irizarry D, Carrillo-Zuniga G, Lee C, Trevino L, et al. Bridging research and policy to address childhood obesity among border Hispanics: a pilot study. Am J Prev Med. 2013;44(3 Suppl 3):S208-14. [PubMed: 23415185].

19. Farzadfar F, Danaei G, Namdaritabar H, Rajaratnam JK, Marcus JR, Khosravi A, et al. National and subnational mortality effects of metabolic risk factors and smoking in Iran: a comparative risk assessment. Popul Health Metr. 2011;9(1):55. [PubMed: 21989074].

20. Kelishadi R, Ardalan G, Gheiratmand R, Gouya MM, Razaghi EM Delavari A, et al. Association of physical activity and dietary behaviours in relation to the body mass index in a national sample of Iranian children and adolescents: CASPIAN Study. Bull World Health Or gan. 2007;85(1):19-26. [PubMed:17242754].

21. W. H. O. Expert Consultation . Appropriate body-mass index for Asian populations and its implications for policy and intervention strategies. Lancet. 2004;363(9403):157-63. [PubMed:14726171].

22. Farzadfar F, Delavari A, Malekzadeh R, Mesdaghinia A, Jamshidi HR, Sayyari A, et al. NASBOD 2013: design, definitions, and metrics. Arch Iran Med. 2014;17(1):7-15. [PubMed: 24444059].

23. Kelishadi R, Hovsepian S, Qorbani M, Jamshidi F, Fallah Z, Djalalinia S, et al. National and sub-national prevalence, trend, and burden of cardiometabolic risk factors in Iranian children and adolescents, 1990 2013. Arch Iran Med. 2014;17(1):71-80. [PubMed: 24444066].

24. Rao S, Simmer K. World Health Organization growth charts for monitoring the growth of Australian children: time to begin the debate.J Paediatr Child Health. 2012;48(2):E84-90. [PubMed: 22050470].

25. de Onis M, Onyango A, Borghi E, Siyam A, Blössner M, Lutter C. Worldwide implementation of the WHO child growth standards. Public Health Nutr. 2012;15(09):1603-10.

26. Ogden CL, Carroll MD, Kit BK, Flegal KM. Prevalence of obesity and trends in body mass index among US children and adolescents, 19992010. JAMA. 2012;307(5):483-90. [PubMed: 22253364].

27. Grummer-Strawn LM, Reinold C, Krebs NF, Centers for Disease C. Use of World Health Organization and CDC growth charts for children aged 0-59 months in the United States. MMWR Recomm Rep. 2010;59(RR-9):1-15. [PubMed: 20829749].

28. Motlagh ME, Kelishadi R, Ardalan G, Gheiratmand R, Majdzadeh R, Heidarzadeh A, et al. Rationale, methods and first results of the Iranian national programme for prevention of chronic diseases from childhood: CASPIAN Study. East Mediterr Health J. 2009;15(2):302-14. [PubMed: 19554976]. 
29. Kelishadi R, Gheiratmand R, Ardalan G, Adeli K, Mehdi Gouya M, Mohammad Razaghi E, et al. Association of anthropometric indices with cardiovascular disease risk factors among children and adolescents: CASPIAN Study. Int J Cardiol. 2007;117(3):340-8. [PubMed: 16860411].

30. Kelishadi R, Heshmat R, Motlagh ME, Majdzadeh R, Keramatian K, Qorbani M, et al. Methodology and Early Findings of the Third Survey of CASPIAN Study: A National School-based Surveillance of Students' High Risk Behaviors. Int J Prev Med. 2012;3(6):394-401. [PubMed: 22783465].

31. Kelishadi R, Majdzadeh R, Motlagh ME, Heshmat R, Aminaee T, Ardalan G, et al. Development and Evaluation of a Questionnaire for Assessment of Determinants of Weight Disorders among Children and Adolescents: The Caspian-IV Study. Int J Prev Med. 2012;3(10):699705. [PubMed: 23112896].

32. Kelishadi R, Ardalan G, Qorbani M, Ataie-Jafari A, Bahreynian M, Taslimi M, et al. Methodology and Early Findings of the Fourth Survey of Childhood and Adolescence Surveillance and Prevention of Adult Non-Communicable Disease in Iran: The CASPIAN-IV Study. Int J Prev Med. 2013;4(12):1451-60. [PubMed: 24498502].

33. Djalalinia S, Kelishadi R, Qorbani M, Peykari N, Kasaeian A, Saeedi Moghaddam S, et al. Suggestions for better data presentation in papers: an experience from a comprehensive study on national and sub-national trends of overweight and obesity. Arch Iran Med. 2014;17(12):830-6. [PubMed: 25481322].

34. Amirkhani A, Motlagh ME. SedaghatM. Health status of Iranian students according to the health-risk behaviors survey 2006-2007. ; .

35. Aazami M, Akbari M, Heshmati B, Alikhani M. The Growth Pattern among Elementary School Students in Kermanshah, Iran. J Isfahan Med Sch. 2012;30(181).

36. Abdollahi AA, Vagari G. The correlation between age, gender and education with obesity in urban population of Golestan province. Iran J Endocrinol Metabol. 2010;12(3):276-82.

37. Ahmadi E, Tehrani AR, Ahmadi A. Prevalence of Obesity, Overweight and Underweight among Elementary School Children in Southern Iran, 2009. Am J Appl Sci. 2010;7(11):1439-42.

38. Akhavan-Karbasi S, Fallah R, Golestan M, Sadr-Bafghi M. Prevalence and risk factors of obesity and overweight among primary school children in Yazd. J Shahid Sadoughi Uni Med Sci Health Serv. 2009;16(5):8-13.

39. Amanolahi A, Sohrabi MR, Montazeri A, Abadi AR, Kolahi AA. Study of obesity levels among school students. Payesh. 2012.

40. Amidimazaheri M, Hoseini M. Prevalence of underweight, overweight and obesity among high school girls in Isfahan. Health System Res. 2010;6(1):1-5.

41. Amini M, Omidvar N, Kimiagar M. Prevalence of overweight and obesity among junior high school students in a district of Tehran.J Res Med Sci. 2007;12(6):315-9.

42. Aminzadeh M, Hosseinzadeh M, Nikfar R, Ghaderian M, Mohsenpourian S. Incidence in Overweight and Obesity among Schoolchildren, Ahvaz-2010. Jundishapur Sci Med J. 2013;12(4).

43. Asadi Noghabi F. Prevalence of obesity and overweight among children in Bandar Abbas. Bimonth J Hormozgan Univ Med Sci. 2011;15(3):218-26.

44. Azimi-Nezhad M, Ghayour-Mobarhan M, Safarian M, Esmailee H, Parizadeh SM, Rajabi-Moghadam M, et al. Anthropometric indices of obesity and the prediction of cardiovascular risk factors in an Iranian population. ScientificWorldJournal. 2009;9:424-30. [PubMed 19526180].

45. Bazhan M, Kalantari N, Ghaffarpoor M, Houshiar-Rad A, Alavi Majd H. Prevalence of obesity, fat distribution and its relationship with food consumption pattern in secondary school girls in Lahijan. Iran J Endocrinol Metabol. 2005;7(1):37-46.

46. Bidad K, Anari S, Tavasoli S, Nazemi L, Gholami N, Zadhush S, et al. Dietary intakes of adolescent girls in relation to weight status. Iran JPublic Health. 2008;37(1):114-8.

47. Baygi F, Dorosty AR, Mehran L. The Prevalence of Childhood Obe- sity and Some Biological-Environmental Associated Factors in Neishabour-Iran, Winter 2006. Iran J DiabObesity. 2010;2(2):7-16.

48. Gargari BP, Behzad MH, Ghassabpour S, Ayat A. Prevalence of overweight and obesity among high-school girls in Tabriz, Iran, in 2001. Food Nutr Bull. 2004;25(3):288-91. [PubMed: 15460273].

49. Gharakhanlou R, Farzad B, Agha-Alinejad H, Steffen LM, Bayati M. Anthropometric measures as predictors of cardiovascular disease risk factors in the urban population of Iran. Arq Bras Cardiol. 2012;98(2):126-35. [PubMed: 22231916].

50. Haeri Behbahani B, Dorosty AR, Eshraghian MR. Assessment of obesity in children: Fat mass index versus body mass index. Tehran Univ Med J. 2009;67(6):408-14.

51. Hajian KA, Sajadi P, Rezvani AR. Prevalence of overweight and underweight among primary school children aged 7-12 years (Babol; 2006). J BabolUni Med Sci. 2008;10(3):83-91.

52. Hajian-Tilaki KO, Sajjadi P, Razavi A. Prevalence of overweight and obesity and associated risk factors in urban primary-school children in Babol, Islamic Republic of Iran. East Mediterr Health J. 2011;17(2):10914. [PubMed: 21735944].

53. Hajian-Tilaki K, Heidari B. Prevalences of overweight and obesity and their association with physical activity pattern among Iranian adolescents aged 12-17 years. Public Health Nutr. 2012;15(12):2246-52. [PubMed: 22578771].

54. Janghorbani M, Parvin F. Prevalence of overweight and thinness in high-school girls in Kerman, Iran. Int J Obes Relat Metab Disord. 1998;22(7):629-33. [PubMed: 9705021].

55. Karandish M, Mohammadpour Ahranjani B, Kalantari N, Eshraghian M, Rashidi MA. Prevalence of overweight and obesity among adolescent students, 1999-2000 in Tehran. Hakim. 2004;7(3):38-43.

56. Kelishadi R, Pour MH, Sarraf-Zadegan N, Sadry GH, Ansari R, Alikhassy $\mathrm{H}$, et al. Obesity and associated modifiable environmental factors in Iranian adolescents: Isfahan Healthy Heart Program - Heart Health Promotion from Childhood. Pediatr Int. 2003;45(4):435-42. [PubMed: 12911481].

57. Khadivzadeh T, Parsai S, Mazloom S. Body mass index and percent of ideal body weight in women of reproductive age in Mashad, 1997-98. J Mashhad Sch Nurs Midwif. 2002;13-14(4):10-8.

58. Maddah M. Overweight and obesity among Iranian female adolescents in Rasht: more overweight in the lower social group. Public Health Nutr. 2007;10(5):450-3. [PubMed: 17411464].

59. Maddah M, Nikooyeh B. Factors associated with overweight in children in Rasht, Iran: gender, maternal education, skipping breakfast and parental obesity. Public Health Nutr. 2010;13(2):196-200. [PubMed: 19545473]

60. Maddah M, Shahraki T, Shahraki M. Underweight and overweight among children in Zahedan, south-east Iran. Public Health Nutr. 2010;13(10):1519-21. [PubMed: 20353615].

61. Mehrkash M, Kelishadi R, Mohammadian S, Mousavinasab F, Qorbani M, Hashemi ME, et al. Obesity and metabolic syndrome among a representative sample of Iranian adolescents. Southeast Asian J Trop Med Public Health. 2012;43(3):756-63. [PubMed: 23077856].

62. Mirhosseini NZ, Yusoff NA, Shahar S, Parizadeh SM, Mobarhen MG, Shakery MT. Prevalence of the metabolic syndrome and its influencing factors among adolescent girls in Mashhad, Iran. Asia Pac J Clin Nutr. 2009;18(1):131-6. [PubMed: 19329406].

63. Mirmiran P, Esmaillzadeh A, Azizi F. Dairy consumption and body mass index: an inverse relationship. Int J Obes (Lond). 2005;29(1):11521. [PubMed: 15534616].

64. Moayeri H, Bidad K, Aghamohammadi A, Rabbani A, Anari S, Nazemi $\mathrm{L}$, et al. Overweight and obesity and their associated factors in adolescents in Tehran, Iran, 2004-2005. Eur J Pediatr. 2006;165(7):489-93. [PubMed: 16718476].

65. Mohammadpour-Ahranjani B, Rashidi A, Karandish M, Eshraghian MR, Kalantari N. Prevalence of overweight and obesity in adolescent Tehrani students, 2000-2001: an epidemic health problem. Public 
Health Nutr. 2004;7(5):645-8. [PubMed: 15251055].

66. Mojibian M, Ghilian Z. Prevalence of obesity among women in Yazd city in 1379-80. J Shahid Sadoughi Uni Med Sci Health Serv. 2002;9(4):3642.

67. Montazerifar F, Karajibani M, Rakhshani F, Hashemi M. Prevalence of underweight, overweight and obesity among high-school girls in Sistan va Baluchistan. East Mediterr Health J. 2009;15(5):1293-300. [PubMed: 20214144].

68. Montazerifar F, Karajibani M, Dashipour A. Prevalence of overweight and obesity and its relation to food intake in middle-school girls in Zahedan. Southern Med J. 1999;1:66-76.

69. Mostafavi H, Dabagh Manesh MH, Zare N. Prevalence of obesity and over weight in adolescents and adult population in Shiraz. Iran J Endocrinol Metabol. 2005;7(1):57-66.

70. Mozaffari $\mathrm{H}, \mathrm{Nabaei}$ B. Obesity and related risk factors. Indian J Pediatr. 2007;74(3):265-7. [PubMed: 17401265].

71. Mozaffari H, Dehghani A, Afkhami M, Rajabiun H. The prevalence of obesity in primary school students in Yazd province. [in Persian]. The 8th Congress of Nutrition in Iran. 2004; .

72. Pourghasem GB, Hamede-Behzad M, Ghassabpour S. Prevalence of overweight and obesity in high-school girls of tabriz. Med J Tabriz Uni Med Sci. 2002;56:34-9.

73. Rafraf M, Gargari BP, Safaiyan A. Prevalence of prehypertension and hypertension among adolescent high school girls in Tabriz, Iran. Food Nutr Bull. 2010;31(3):461-5. [PubMed: 20973466].

74. Rashidi A, Mohammadpour-Ahranjani B, Karandish M, Vafa M, Hajifaraji M, Ansari F, et al. Obese and female adolescents skip breakfast more than their non-obese and male peers. Open Med. 2007;2(4).

75. Sadeqipoor H, Jazaeri A, Nikbin H, Eshraqian M. Study of weight, height, body mass index, energy and nutrients intake of 11-14 years old girls, Tehran. Tehran UnivMed J. 1999;57(4):63-9.

76. Salem Z, Vazirinejad R. Prevalence of obesity and metabolic syndrome in adolescent girls in South East of Iran. PakJ Med Sci. 2009;25(2):196200.

77. Salem Z. Anthropometric characteristics of Rafsanjan primary schoolchildren based on body mass index and waist circumference in 2008. J Kerman Uni Med Sci. 2011;18(1):40-8.

78. Saffari F, Esmailzadehha N, Shariatinejad K. Anthropometric indices in 6-14 year-old girls in Qazvin, Iran. J Isfahan Med School. 2012;29(165):1-11.

79. Sohailifar J, Sadri G. Prevalence of obesity in hamadan primaryschools'students in 1998. Sci J Hamadan Uni Med Sci Health Serv. 2000;7(2):15-9.

80. Sotoodeh G, Lesan S. A sutdy of anthropometric indices and determination of the effective factors on them in Hassanabad Khaleseh of Eslamshahr, 1373. Tehran Uni Med J. 1997;55(3):84-9.

81. Taheri F, Kazemi T. Prevalence of overweight and obesity in 7 to 18 yearold children in Birjand/Iran. ranJ Pediatr. 2009;19(2):135-40.

82. Taheri F, Kazemi T, Chahkandi T, Namakin K, Zardast M, Bijari B. Prevalence of overweight, obesity and central obesity among elementary school children in Birjand, east of Iran, 2012. J Res Health Sci. 2013;13(2):157-61. [PubMed: 24077473].

83. Veghari G, Sedaghat M, Joshaghani H, Hoseini A, Niknezhad F, Angizeh $\mathrm{A}$, et al. The prevalence of obesity and its related risk factor in the north of iran in 2006. J Res Health Sci. 2010;10(2):116-21. [PubMed: 22911934].
84. Veghari G, Sedaghat M, Banihashem S, Moharloei P, Angizeh A, Tazik $\mathrm{E}$, et al. Trends in waist circumference and central obesity in adults, northern iran. Oman Med J. 2012;27(1):50-3. [PubMed: 22359726].

85. Kelishadi R, Ardalan G, Gheiratmand R, Majdzadeh R, Hosseini M, Gouya MM, et al. Thinness, overweight and obesity in a national sample of Iranian children and adolescents: CASPIAN Study. Child Care Health Dev. 2008;34(1):44-54. [PubMed: 18171443].

86. Khashayar P, Heshmat R, Qorbani M, Motlagh ME, Aminaee T, Ardalan G, et al. Metabolic Syndrome and Cardiovascular Risk Factors in a National Sample of Adolescent Population in the Middle East and North Africa: The CASPIAN III Study. Int J Endocrinol. 2013;2013:702095. [PubMed: 23476647].

87. Hosseini-Esfahani F, Mousavi Nasl Khameneh A, Mirmiran P, Ghanbarian A, Azizi F. Trends in risk factors for cardiovascular disease among Iranian adolescents: the Tehran Lipid and Glucose Study, 1999-2008.J Epidemiol. 2011;21(5):319-28. [PubMed: 21804294].

88. Janghorbani M, Amini M, Willett WC, Mehdi Gouya M, Delavari A, Alikhani S, et al. First nationwide survey of prevalence of overweight, underweight, and abdominal obesity in Iranian adults. Obesity (Silver Spring). 2007;15(11):2797-808. [PubMed: 18070771].

89. Chan M. From new estimates to better data. Lancet. 2012;380(9859):2054. [PubMed: 23245596].

90. Tranfield D, Denyer D, Smart P. Towards a Methodology for Developing Evidence-Informed Management Knowledge by Means of Systematic Review. Brit J Manag. 2003;14(3):207-22.

91. Naghavi M, Abolhassani F, Pourmalek F, Lakeh M, Jafari N, Vaseghi S, et al. The burden of disease and injury in Iran 2003. Popul Health Metr. 2009;7:9. [PubMed: 19527516].

92. Esteghamati A, Meysamie A, Khalilzadeh O, Rashidi A, Haghazali M, Asgari F, et al. Third national Surveillance of Risk Factors of NonCommunicable Diseases (SuRFNCD-2007) in Iran: methods and results on prevalence of diabetes, hypertension, obesity, central obesity, and dyslipidemia. BMC Public Health. 2009;9:167.[PubMed: 19480675]

93. Asgari F, Aghajani H, Haghazali M, Heidarian H. Non-communicable diseases risk factors surveillance in Iran. Iran J Public Health. 2009;38(1):119-22.

94. Kasaeian A, Eshraghian MR, Rahimi Foroushani A, Niakan Kalhori SR, Mohammad K, Farzadfar F. Bayesian autoregressive multilevel modeling of burden of diseases, injuries and risk factors in Iran 1990 - 2013. Arch Iran Med. 2014;17(1):22-7. [PubMed: 24444061].

95. Parsaeian M, Farzadfar F, Zeraati H, Mahmoudi M, Rahimig hazikalayeh G, Navidi I, et al. Application of spatio-temporal model to estimate burden of diseases, injuries and risk factors in Iran 1990 2013. Arch Iran Med. 2014;17(1):28-33. [PubMed: 24444062].

96. Djalalinia S, Peykari N, Qorbani M, Moghaddam SS, Larijani B, Farzadfar F. Obesity Researches Over the Past 24 years: A Scientometrics Study in Middle East Countries. Int J Prev Med. 2015;6:38. [PubMed: 26015861].

97. Sarrafzadegan N, Kelishadi R, Esmaillzadeh A, Mohammadifard N, Rabiei K, Roohafza $\mathrm{H}$, et al. Do lifestyle interventions work in developing countries? Findings from the Isfahan Healthy Heart Program in the Islamic Republic of Iran. Bull World Health Organ. 2009;87(1):3950. [PubMed: 19197403].

98. Reilly JJ, Kelly J, Wilson DC. Accuracy of simple clinical and epidemiological definitions of childhood obesity: systematic review and evidence appraisal. Obes Rev. 2010;11(9):645-55. [PubMed: 20059704]. 